\title{
Origin of calcium in pedogenic carbonate nodules from silicate watersheds in the Far North Region of Cameroon: Respective contribution of in situ weathering source and dust input
}

\author{
Fabienne Dietrich $^{\mathrm{a}, *}$, Nathalie Diaz ${ }^{\mathrm{a}}$, Pierre Deschamps ${ }^{\mathrm{b}}$, Benjamin Ngounou Ngatcha ${ }^{\mathrm{c}}$, \\ David Sebag, $^{\mathrm{a}, \mathrm{e}}$, Eric P. Verrecchia ${ }^{\mathrm{a}}$ \\ ${ }^{a}$ Earth Surface Dynamics Institute, Lausanne University, Switzerland \\ b Aix-Marseille Univ, CNRS, IRD, CEREGE, Europole Méditerranéen de l'Arbois, BP80, 13545 Aix-en-Provence, France \\ c Earth Science Department, LMI Picass'Eau, Ngaoundere University, Ngaoundere, Cameroon \\ d Normandie Univ, UNIROUEN, UNICAEN, CNRS, M2C, 76000 Rouen, France \\ e HSM, LMI Picass'Eau, IRD, Université de Montpellier, Montpellier, France
}

\section{A R T I C L E I N F O}

\section{Keywords:}

Pedogenic carbonate

Ca sources

Strontium and neodymium isotopes

Weathering

Vertisols

\begin{abstract}
A B S T R A C T
Significant amounts of pedogenic Ca-carbonate nodules have been observed in the Far North Region of Cameroon in the carbonate-free watershed of the Mayo Tsanaga, thus a priori not favourable for carbonate nodules accumulations. These nodules are associated with a Clay-Rich Parent Material (CRPM), covering either a granitic (upstream) or a greenstone bedrock (downstream). In this peculiar context, the amount of pedogenic carbonate nodules represents large quantities of $\mathrm{Ca}$ and $\mathrm{C}$. Therefore, determining the Ca sources for pedogenic carbonate nodules is a key point regarding the mechanisms leading to carbonate nodule precipitation and their role in the biogeochemical cycle of Ca. Three sites, two on granite and one on greenstone, were studied by combining $\mathrm{Sr}$ et $\mathrm{Nd}$ isotope systematics in order to assess the Ca sources of carbonate nodules and the origin of the CRPM associated with nodules.

Carbonate nodules have a distinct $\mathrm{Sr}$ isotopic composition in each profile, pointing to the contribution of a local source for $\mathrm{Ca}$. Sr isotopic compositions of plagioclases and carbonate nodules display a concomitant variation in each profile, indicating that plagioclases acted as a $\mathrm{Sr}$, and thus $\mathrm{Ca}$, source for the carbonate nodules. Nevertheless, carbonate nodules have a higher Sr isotopic signature than plagioclases, implying the contribution of another more radiogenic $\mathrm{Sr}$ source. $\mathrm{Sr}$ and $\mathrm{Nd}$ isotope data from the CRPM show that it is a mixture of weathered bedrock compounds and Saharan dust. This result highlights two other potential Sr and Ca sources: biotite and Saharan dust. Calculations of their respective contributions demonstrate that in situ weathering significantly adds to the Ca source of nodules. This result contrasts with many other studies from similar settings that highlight the importance of allochthonous - marine, aeolian - inputs. This conclusion points out that a substantial part of $\mathrm{Ca}$ is transferred from the local bedrock to the carbonate nodules. This mechanism seems to have been possible as peculiar edaphic conditions preserved the primary Ca sources, making in situ weathering a significant process for providing Ca.
\end{abstract}

\section{Introduction}

Pedogenic carbonates are ubiquist features on continents and they mainly result from interactions between the calcium (Ca) and carbon (C) terrestrial cycles (Lal et al., 1999). In silicate peculiar settings, pedogenic carbonate can act as C trapping systems (e.g Schlesinger, 1982; Khadkikar et al., 1998; Dart et al., 2007; Zamanian et al., 2016). C in pedogenic carbonate mainly stems from soil respiration (Cerling, 1984; Verrecchia, 2011; Hasinger et al., 2015), but the origin of Ca differs depending on the geochemical context. Generally, two main $\mathrm{Ca}$ sources are identified, either lithogenic (rock weathering, e.g. Durand et al., 2006) or atmospheric (e.g. Chiquet et al., 1999). As determining the $\mathrm{Ca}$ origin of pedogenic carbonate is crucial to infer how and when pedogenic carbonates act as a potential C sink (Elbersen et al., 2000), study Ca sources remains relevant. Such studies have been widely documented from various geological settings, especially in the arid/ semi-arid environments where such features are relatively abundant (e.g. Capo and Chadwick, 1999; Hamidi et al., 2001; Van der Hoven

\footnotetext{
* Corresponding author.

E-mail address: fabienne.dietrich@unil.ch (F. Dietrich).
} 


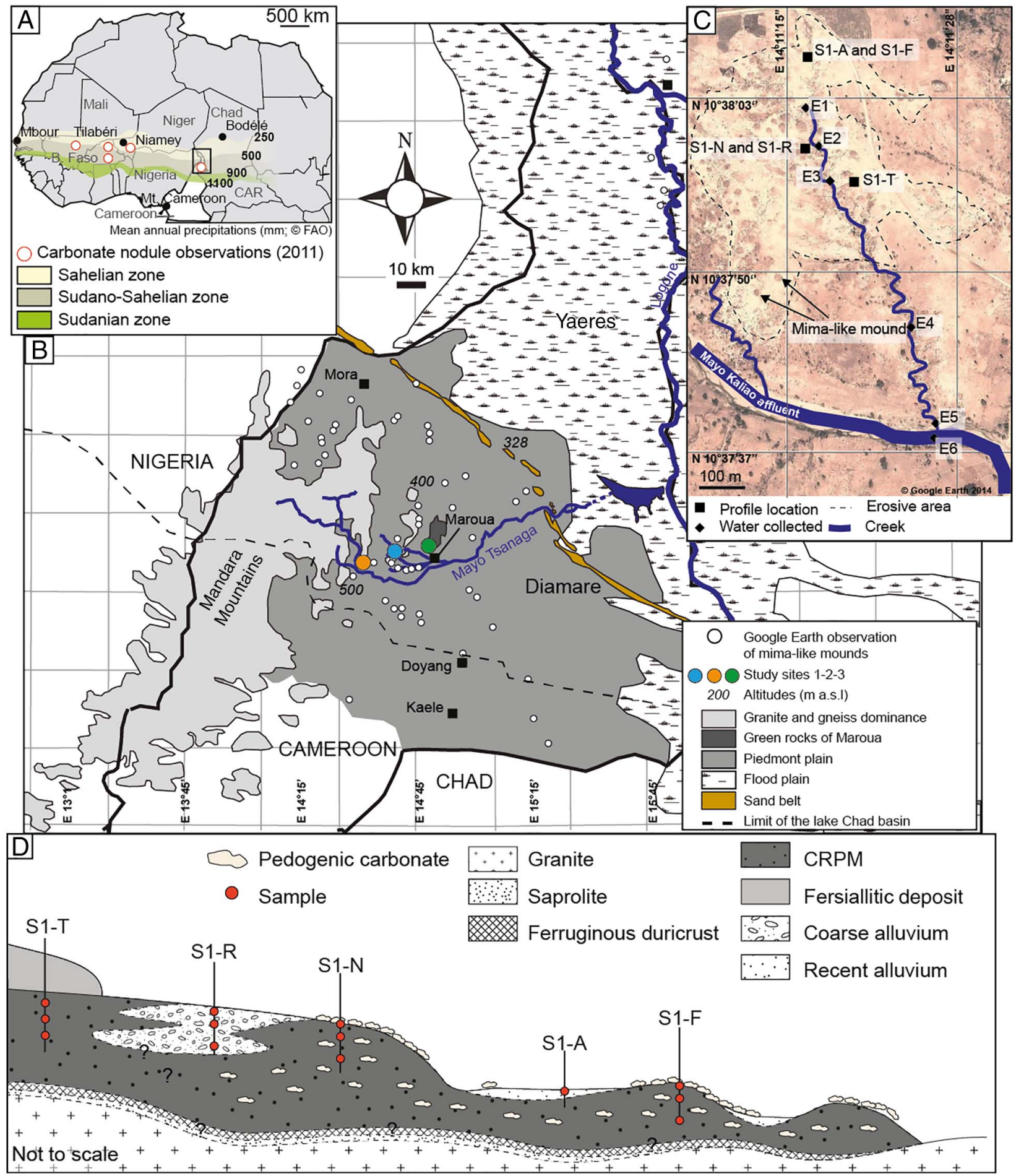

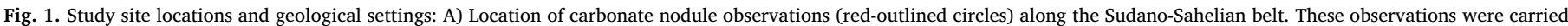

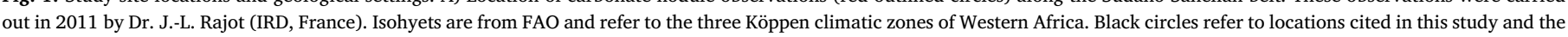

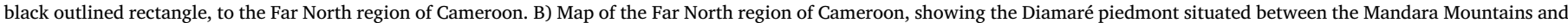

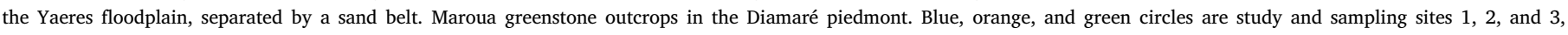

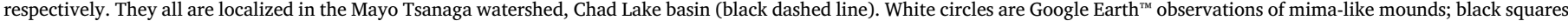

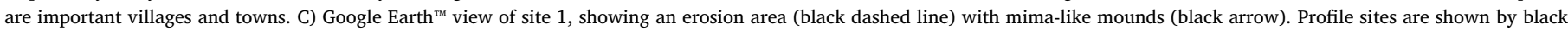

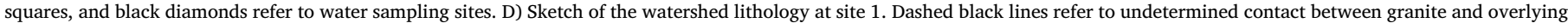
sediments. (For interpretation of the references to color in this figure legend, the reader is referred to the web version of this article.)

and Quade, 2002; Violette et al., 2010).

The Sudano-Sahelian belt gives remarkable examples of pedogenic carbonate settings, where large amounts of Ca-carbonate nodules are observed in carbonate-free watersheds (Fig. 1A). The origin of these carbonates remains puzzling since carbonate-free watersheds are a priori not favourable to accumulations of Ca-carbonate nodules. First, $\mathrm{Ca}$ is usually not very abundant, especially in granitic settings (Bowen, 1979). Second, Ca is easily leached from primary and secondary minerals (Likens et al., 1998), especially in subtropical environments, where rates of chemical weathering are high (White and Blum, 1995), 
leading to depletion of alkali and alkaline earth cations in soils. Consequently, in such lithogenic and geographic settings, Ca-carbonate nodule occurrence is not expected as Ca should not be available for their formation.

In the Far North Region of Cameroon, pedogenic carbonate nodules have been observed in a silicate watershed. They are widespread, well developed, and have been described numerous times, without any clear explanation of their genesis (Martin, 1961; Sieffermann, 1967; Brabant and Gavaud, 1985; Morin, 2000, Fig. 1B). Carbonate nodules are associated with a soil clay-rich parent material, in which the nodules have precipitated. The origin of this soil clay-rich parent material is also an unresolved issue, as several authors suggested that a significant amount of Saharan dust contributed to the soil composition in this area (Mpeck, 1994; Lamotte et al., 1997).

Consequently, the aim of this study is (1) to identify the Ca sources of the pedogenic carbonate nodules from the Far North Region of Cameroon, and (2) to document the origin of the host soil parent material. Strontium isotopes $\left({ }^{87} \mathrm{Sr} /{ }^{86} \mathrm{Sr}\right)$ were used to identify the $\mathrm{Ca}$ sources, since they are considered as a suitable tool to trace $\mathrm{Ca}$ provenance and mobility (Capo et al., 1998). Such measurements can be combined with neodymium (Nd) isotope systematics to determine the origin of sediments (McLennan et al., 1993) and trace aeolian contributions in sediments (Grousset et al., 1998; Grousset and Biscaye, 2005) and soils (Borg and Banner, 1996; Dia et al., 2006). In this study, $\mathrm{Nd}$ isotopes are used to document the origin of the soil parent material in which the nodules precipitated. In addition to the identification of the different sources of element/material of the system, such a multiisotopic approach can provide quantitative estimations of the respective contributions of the different $\mathrm{Ca}$ reservoirs. This qualitative and quantitative study of $\mathrm{Ca}$ and sediment origin of soils aims to provide some insights into the processes at the origin of the formation of Cacarbonate nodules, which remain unexplained in this unfavourable chemical and geographical context.

\section{General settings}

Study sites are located at the foot of the Mandara Mountains (Diamare piedmont), in the watershed of the Mayo Tsanaga and the sub-watershed of the Mayo Kaliao (Fig. 1B). These two Mayos ("Mayo" in local Fufulde language means "intermittent stream") are tributaries of the Logone River, which flows to the Lake Chad endorheic basin (Olivry and Naah, 1999). The average annual rainfall is $800 \mathrm{~mm}$ and the average temperature is $27.6{ }^{\circ} \mathrm{C}$ at Salak weather station (Suchel, 1988). The year is divided into a dry season, from October to May, and a wet season, from June to September (Suchel, 1988). During the dry season, the dry Harmattan wind blows from north to southwest, loaded with dust likely originating from the Bodélé depression (Kalu, 1979; Schwanghart and Schütt, 2008).

The Mandara Mountains are mainly composed of Precambrian anatexis granite and gneiss (Gazel et al., 1956). Downstream, Tertiary metamorphic rocks of the green schist facies, known as "greenstones of Maroua", outcrop northern Maroua (Brabant and Gavaud, 1985). Various sedimentary deposits compose the Diamaré piedmont. They resulted from numerous sedimentological cycles related to climate variations that occurred during the Quaternary in the Far North Region of Cameroon (Hervieu, 1967). The pediment is mainly composed of quartz and feldspar minerals with a decreasing grain size distribution towards the Yaeres floodplain. This pediment interfingers with a clayrich sediment and a coarse alluvium (Hervieu, 1967; Morin, 2000). The saprolite, developed from the granitic bedrock, is covered by a clay-rich sediment associated with sand lenses and occasionally by a ferruginous duricrust (Morin, 2000). A clay-rich sediment also covers the greenstones of Maroua downstream.

Carbonate nodules are pedogenic in origin and probably formed between 11.0 and $5.0 \mathrm{ka}$ cal BP $(\mathrm{n}=19$, radiocarbon dating from Diaz et al., 2016b). They are thus inherited from a humid period (Diaz et al., 2016a, 2016b) called the African Humid Period (from 14.8 to 5.5 ka BP, de Menocal et al., 2000). They are observed within or at the surface of a clay-rich sediment, called hereafter Clay-Rich Parent Material (CRPM). The CRPM is widespread in the Far North Region of Cameroon and has already been amply described in the literature (Erhart, 1954; Pias, 1962; Sieffermann, 1967; Bocquier, 1973; Brabant and Gavaud, 1985; Morin, 2000). Allochthonous contributions, such as aeolian input with some reworking after deposition, have been proposed to explain the large extension of the CRPM (Maley, 1981; Mpeck, 1994; Diaz et al., 2016a). The CRPM is observed with various morphologies in the landscape, such as non-anthropologic earth mounds or whale backs (Diaz et al., 2016a). The earth-mounds developed in the CRPM in highly eroded areas (easily observed with Google Earth ${ }^{\mathrm{TM}}$, Fig. 1C) have been defined as mima-like mounds (Cramer and Barger, 2014; Diaz et al., 2016a). Diaz et al. (2016a, 2016b) proposed that mima-like mounds from the Far North region of Cameroon resulted from the degradation of Vertisols, developed in the CRPM during the African Humid Period. Carbonate nodules are a common Vertisol feature (Wieder and Yaalon, 1982; Kovda et al., 2006). Due to their darker colour and their flatter morphologies, Vertisols are less degraded downstream in the greenstone area (Brabant and Gavaud, 1985; Diaz et al., 2016a). The landscape changes drastically in this zone. Relief is composed of green hills, on which fersiallitic soils form along the slopes. At the foot of these hills, where the slopes become gentler, Vertisols develop in the CRPM, but are partly eroded by gullies (Fig. 2 down, Brabant and Gavaud, 1985).

\section{Materials and methods}

\subsection{Study sites}

Three sites with abundant carbonate nodules have been selected for this study (Fig. 1B). Two are associated with granitic bedrock and one with a greenstone. An exhaustive field description of soils can be found in Diaz et al. (2016a) for site 1 and 2 and is summarized as follows. In site 1, the CRPM is observed either i) in contact with a coarse alluvium, ii) covered by a fersiallitic deposit, or iii) directly overlying granite inselbergs (Fig. 2). Recent alluvium, composed of reworked CRPM, circles mima-like-mounds (Fig. 2). Five profiles (S1-F, S1-N, S1-R, S1-A and S1-T) were sampled (Diaz et al., 2016a; Fig. 1C and D) in order to have a representative view of the different sedimentary deposits observed. S1-F and S1-N are in the CRPM, the first one being located at the centre of a mima-like mound and the second interfingering with the coarse alluvium. CRPM in these two profiles has a clay-loamy texture and a massive structure. The skeleton (i.e. soil fraction $>2$ $\mathrm{mm}$ ) can reach $20 \%$ of the soil volume and is mainly composed of carbonate nodules. The soil $\mathrm{pH}$ varies between 8.7 and 9.6.

CRPM includes sub-millimetric and millimetric disorthic carbonate and Fe-Mn oxide nodules (disorthic means that nodules contain grains from the surrounding soil matrix, but are partially reworked within the soil profile; Wieder and Yaalon, 1982; Fig. 3A). In thin section, the fine fraction is composed of calcite and clays, and the coarse fraction of multi-sized silicate grains (mainly quartz, plagioclases, and K-feldspar). Carbonate nodules are composed of a micritic matrix (Fig. 3B), in which two different distribution patterns are observed. The first pattern is random and defined as an open-porphyric coarse-fine related distribution (grains are mainly quartz, plagioclases, and K-feldspar). The second pattern is fan-like and includes much less silicate grains. Septarian cracks are partially infilled or coated with sparitic calcite.

Profile S1-R contains only small carbonate nodules $(<1 \mathrm{~cm})$ and is composed by a silicate coarse alluvium interfingering with the CRPM. Profile S1-A refers to a border of a mima-like mound and is made of a recent fine alluvium, any carbonate nodules have been observed in this profile. Finally, profile S1-T is a buried CRPM layer, under a fersiallitic deposit, without carbonate nodules.

Site 2 is situated upstream of site 1 in similar settings. This site was 


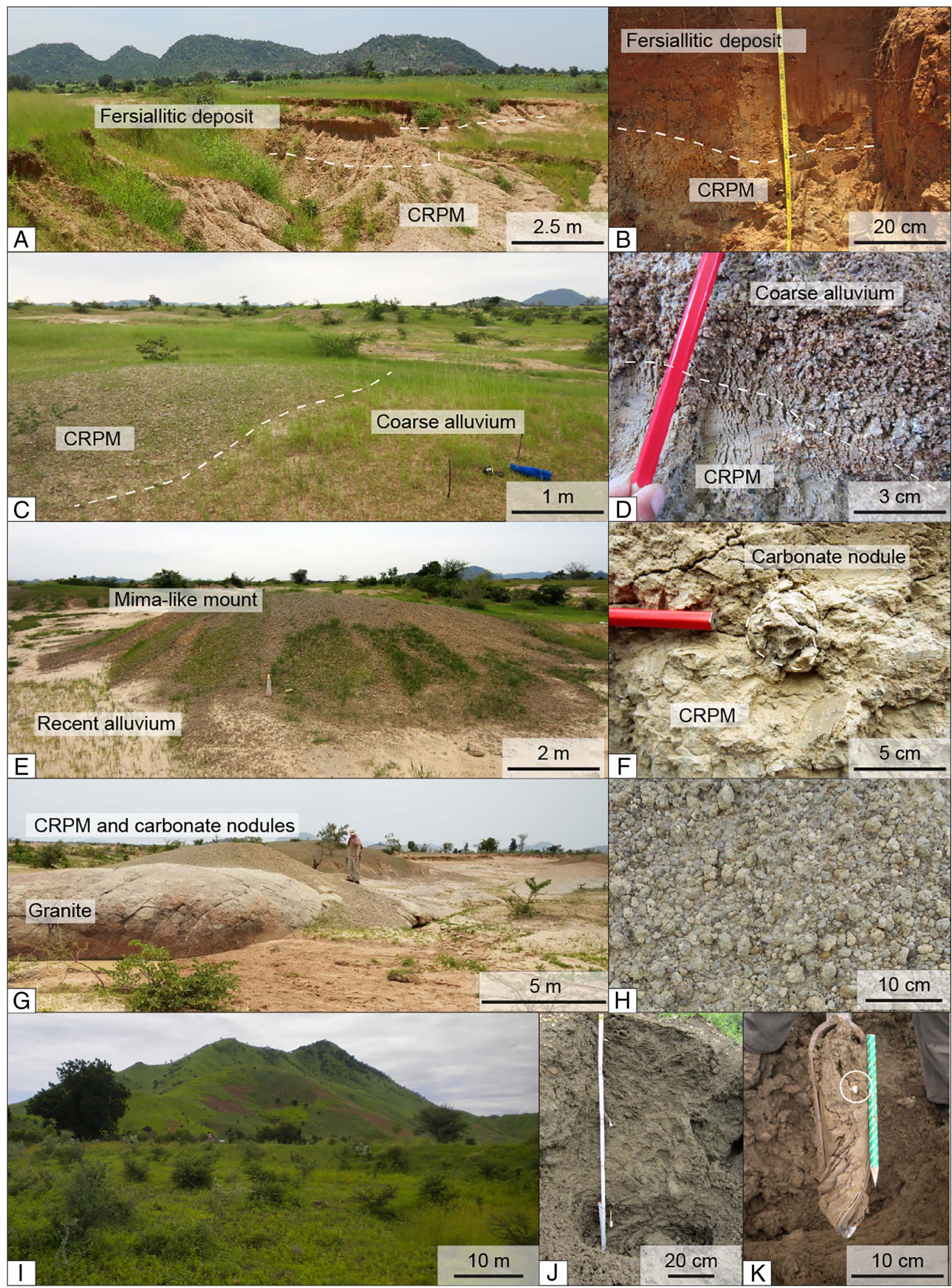

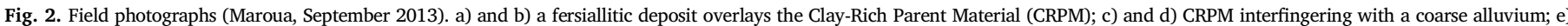

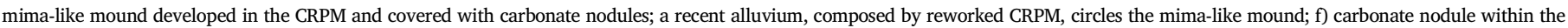

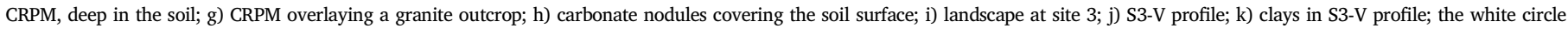
highlights a fragment of a carbonate nodule. 

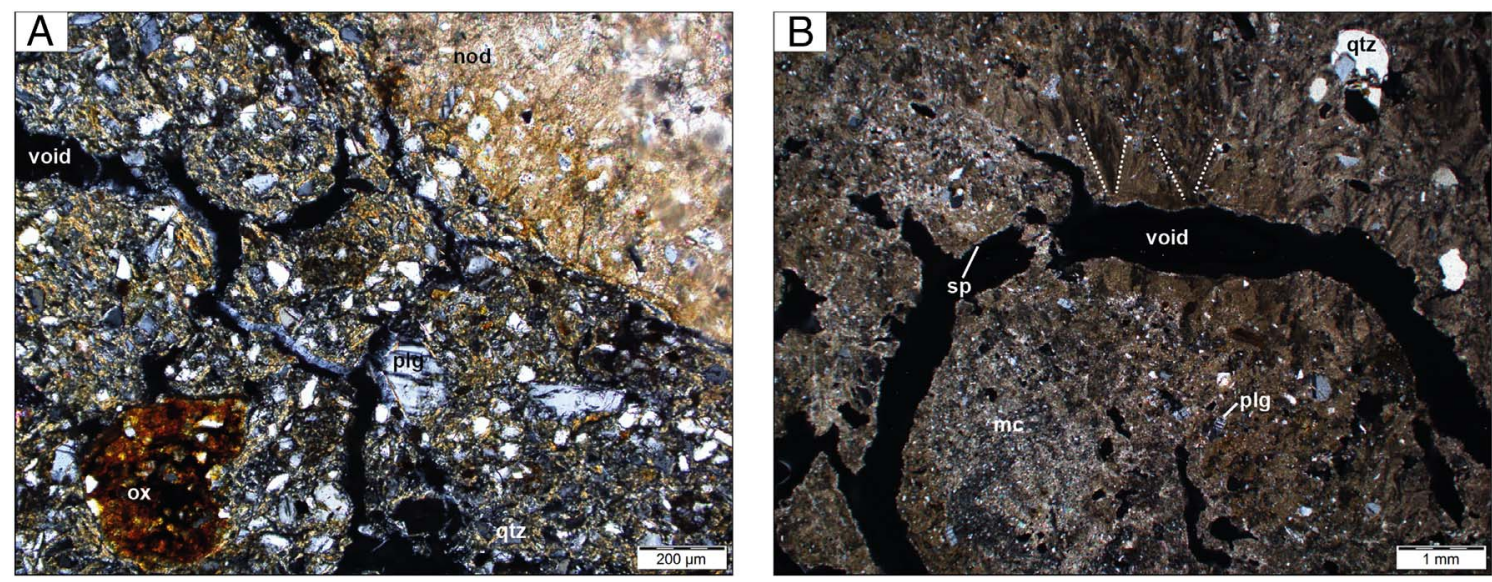

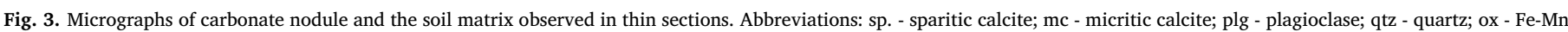

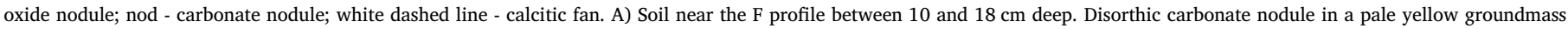

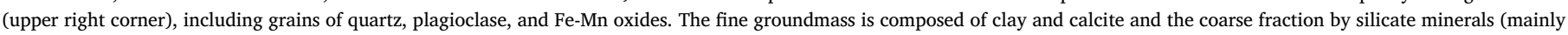

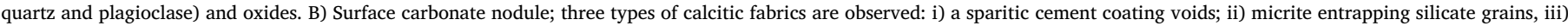
fan-like cements. (For interpretation of the references to color in this figure legend, the reader is referred to the web version of this article.)

sampled in order to evaluate the potential spatial heterogeneity of the isotopic compositions of both the carbonate nodules and the CRPM. One soil profile, S2-S, was sampled in the centre of a mima-like mound and has the same characteristics as S1-F and S1-N.

Site 3 is located downstream of site 1 and 2, where the Maroua greenstone outcrops (Fig. 1B, Fig. 2 down). This third site was selected to assess the variability of the CRPM composition and the potential influence of different bedrocks. The soil profile (S3-V) has a microgranular structure in its upper horizon and a sub-angular blocky to massive structure in its deeper horizons. Slickensides are observed all along the soil profile. Carbonate nodules and silicate gravels compose the soil skeleton $(<10 \%$ vol). Small Fe-Mn oxide nodules are also present. The texture is clayey and the $\mathrm{pH}$ varies between 7.8 and 8.9. The organic matter content is very low in all the studied profiles whatever the sites (TOC $<0.5 \%$ ).

\subsection{Collection and preparation of samples}

Samples were collected at various depths, i.e. 5,20 , and $60 \mathrm{~cm}$ in S1-R, S1-N, S1-F, S2-S, and S3-V profiles. Depths of sampling were selected as follows for S1-T profile: $40,60,80 \mathrm{~cm}$. S1-A, the recent alluvium, has been sampled in its first $5 \mathrm{~cm}$. Nodules have also been collected at the surface of S1-N, S1-F, S2-S, and S3-V profiles. Granite (GR) from inselbergs was also gathered as well as waters from intermittent streams (E1 to E6 in Fig. 1C). The greenstone of Maroua (RV) was also sampled at site 3. The bulk soil fraction (i.e. fine earth $<2 \mathrm{~mm}$ ) and the nodule fraction were analysed separately in each sample. Soil samples were sieved at $2 \mathrm{~mm}$ (fine earth) and crushed in an agate bowl, as well as nodules and rocks. Water samples were filtered at $2 \mu \mathrm{m}$ (with Poly Tetra Fluor Ethylene - PTFE - filters), acidified at $\mathrm{pH} 1$ with ultrapure $\mathrm{HNO}_{3}$ directly in the field, and stored in fluorinated ethylene propylene bottles.

Regarding Sr isotopes, it is recommended to consider a ${ }^{87} \mathrm{Sr} /{ }^{86} \mathrm{Sr}$ ratio for each mineral separately, instead of bulk rock, as each mineral has a distinct ${ }^{87} \mathrm{Sr} /{ }^{86} \mathrm{Sr}$ ratio and weathers at various rates (Clauer and Chaudhuri, 1995; Blum and Erel, 1997). At sites 1 and 2, the granitic bedrock contains Ca mainly in plagioclases. At site 3, the greenstone bedrock includes plagioclases and epidotes, which both contain $\mathrm{Ca}$. Since epidote is much less abundant and less enriched in $\mathrm{Ca}$ and $\mathrm{Sr}$ than plagioclase, the focus will be on plagioclases. However, as biotite usually has a high ${ }^{87} \mathrm{Sr} /{ }^{86} \mathrm{Sr}$ ratio due to its high $\mathrm{Rb} / \mathrm{Sr}$ ratio (Faure and Powell, 2012), it can contribute to some radiogenic Sr incorporated into carbonate nodules. Therefore, biotite was also analysed. Biotite and plagioclases from granite, greenstone, and soil samples (S1-F8, S1-N8 and S-20) were separated using heavy liquor (Sodium PolyTungstate, SPT) and hand checked with a binocular. Samples were washed several times with milliQ-water and leached with $3 \mathrm{~N} \mathrm{HCl}$ to remove any trace of heavy liquor and impurities.

\subsection{Analytical methods}

Carbonate contents of soil fine earth $(<2 \mathrm{~mm})$ and nodules were determined using the mass loss method with $2.2 \mathrm{M}$ acetic acid (using $2 \mathrm{~g}$ of material). The analyses were performed in triplicates on five samples in order to validate the method. Diversity and relative contents of minerals were determined using X-ray diffraction analysis (XRD) on bulk samples using an ARL Xtra diffractometer (Thermo) at Lausanne University. Peak intensities of major minerals were converted into relative abundances, organic matter and amorphous phases being included in an undetermined fraction. Results for carbonate contents and mineralogical compositions are given in Table 1.

Geochemical analyses were performed in a clean laboratory (class 1000) using bi-distilled acid. Two fractions from both soils and nodules were analysed: i) the carbonate phase, obtained after a $2.2 \mathrm{M}$ acetic acid leaching (Arunchalam et al., 1996), and ii) a residue phase (obtained after acid acetic extraction) dissolved using 2:1 $\mathrm{HNO}_{3}: \mathrm{HF}$. Finally, the granite (GR), plagioclases, biotite, and greenstone of Maroua (RV) were analysed as a bulk fraction after total dissolution in 1:1 $\mathrm{HNO}_{3}: \mathrm{HF}$. Water samples were evaporated and recovered with $\mathrm{HNO}_{3} 1 \mathrm{M}$. Protocols and potential associated errors are given in Supplementary materials (Appendix A).

Sr content was separated using Sr spec resin with $1 \mathrm{~N} \mathrm{HNO}_{3}$ and $\mathrm{Nd}$ using TRU spec resin with $1 \mathrm{~N} \mathrm{HNO}_{3}$ and $\mathrm{Ln}$ resin with $0.5 \mathrm{~N} \mathrm{HCl}$ following a procedure modified from Pin et al. (1994). Isotope analyses were performed with a NEPTUNE + Multi-Collector ICP-MS at Geneva University for $\mathrm{Sr}$ and $\mathrm{Nd}$ isotopes and at CEREGE (Aix-Marseille, France) for $\mathrm{Sr}$ isotopes as well. The analytical reproducibility for $\mathrm{Sr}$ obtained during the course of this study on the SRM987 standard in Geneva and NBS-981 standard in CEREGE was $50 \mathrm{ppm}(2 \sigma)$. The analytical reproducibility for $\mathrm{Nd}$ obtained during the course of this study on the Jndi-1 standard was $20 \mathrm{ppm}(2 \sigma) . \varepsilon(\mathrm{Nd})$ is normalized to CHondritic Uniform Reservoir (CHUR $-{ }^{144} \mathrm{Nd} /{ }^{143} \mathrm{Nd}=0.512638$; Jacobsen and Wasserburg, 1980):

$\varepsilon(\mathrm{Nd})=\left(\frac{\left(\frac{{ }^{143} \mathrm{Nd}}{{ }^{144} \mathrm{Nd}}\right)_{\text {sample }}}{\left(\frac{143 \mathrm{Nd}}{{ }^{144} \mathrm{Nd}}\right)_{\mathrm{CHUR}}}-1\right) \times 10^{4}$ 
Table 1

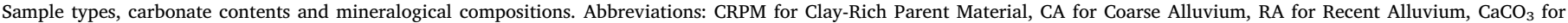
carbonate content, Phyllo for phyllosilicates, Qtz for quartz, Fdp for feldspar, Plg for plagioclase, Cc for calcite, Dlt for dolomite, Gth for goethite, Akt for ankerite.

\begin{tabular}{|c|c|c|c|c|c|c|c|c|c|c|c|c|c|c|c|}
\hline \multirow[t]{2}{*}{ Samples } & \multirow[t]{2}{*}{ Site } & \multirow[t]{2}{*}{ Materials } & & \multirow[t]{2}{*}{ Profile } & \multirow[t]{2}{*}{ Depth cm } & \multirow[t]{2}{*}{ Carbonate $\%$} & \multicolumn{9}{|c|}{ Mineralogy \% } \\
\hline & & & & & & & Phyllo & Qtz & Fdp & $\mathrm{Plg}$ & Cct & Dlt & Gth & Akt & Undosed \\
\hline S1-FSNO1 & 1 & CRPM & Nodule & S1-F & 0 & 77.5 & 14 & 10 & 3 & 0 & 67 & 0 & 0 & 0 & 5 \\
\hline S1-FSNO2 & 1 & CRPM & Nodule & S1-F & 0 & 72.5 & 14 & 10 & 3 & 6 & 62 & 0 & 0 & 0 & 5 \\
\hline S1-F1NO & 1 & CRPM & Nodule & S1-F & 5 & 74.0 & n.a. & n.a. & n.a. & n.a. & n.a. & n.a. & n.a. & n.a. & n.a. \\
\hline S1-F4NO & 1 & CRPM & Nodule & S1-F & 20 & 77.0 & n.a. & n.a. & n.a. & n.a. & n.a. & n.a. & n.a. & n.a. & n.a. \\
\hline S1-F8NO & 1 & CRPM & Nodule & S1-F & 60 & 68.0 & n.a. & n.a. & & n.a. & n.a. & n.a. & n.a. & n.a. & n.a. \\
\hline S1-F1SO & 1 & CRPM & Soil & S1-F & 5 & 3.0 & 29 & 34 & 21 & 9 & 3 & 1 & 0 & 0 & 2 \\
\hline S1-F4SO & 1 & CRPM & Soil & S1-F & 20 & 4.0 & 25 & 45 & 16 & 4 & 3 & 1 & 0 & 0 & 6 \\
\hline S1-F8SO & 1 & CRPM & Soil & S1-F & 60 & 1.0 & 26 & 43 & 9 & 6 & 1 & 1 & 2 & 0 & 11 \\
\hline S1-NSNO1 & 1 & CRPM & Nodule & S1-N & 0 & 71.5 & 16 & 16 & 6 & 0 & 54 & 0 & 0 & 0 & 8 \\
\hline S1-NSNO2 & 1 & CRPM & Nodule & S1-N & 0 & 69.0 & 10 & 8 & 16 & 16 & 46 & 0 & 0 & 0 & 4 \\
\hline S1-NSNO3 & 1 & CRPM & Nodule & S1-N & 0 & 69.5 & 11 & 12 & 6 & 8 & 57 & 0 & 0 & 0 & 6 \\
\hline S1-N1NO & 1 & CRPM & Nodule & S1-N & 5 & 72.5 & n.a. & n.a. & n.a. & n.a. & n.a. & n.a. & n.a. & n.a. & n.a. \\
\hline S1-N4NO & 1 & CRPM & Nodule & S1-N & 20 & 75.5 & n.a. & n.a. & n.a. & n.a. & n.a. & n.a. & n.a. & n.a. & n.a. \\
\hline S1-N8NO & 1 & CRPM & Nodule & S1-N & 60 & 70.5 & n.a. & n.a. & n.a. & n.a. & n.a. & n.a. & n.a. & n.a. & n.a. \\
\hline S1-N1SO & 1 & CRPM & Soil & S1-N & 5 & 1.5 & 18 & 41 & 20 & 7 & 2 & 9 & 0 & 0 & 2 \\
\hline S1-N4SO & 1 & CRPM & Soil & S1-N & 20 & 2.5 & 20 & 50 & 19 & 6 & 2 & 0 & 0 & 0 & 1 \\
\hline S1-N8SO & 1 & CRPM & Soil & S1-N & 60 & 2.0 & 30 & 35 & 10 & 20 & 2 & 1 & 0 & 0 & 2 \\
\hline S1-R0SO & 1 & $\mathrm{CA}$ & Soil & S1-R & 5 & 1.0 & 16 & 38 & 18 & 10 & 7 & 5 & 5 & 0 & 1 \\
\hline S1-R3SO & 1 & $\mathrm{CA}$ & Soil & S1-R & 20 & 0.5 & 14 & 58 & 17 & 8 & 1 & 1 & 0 & 0 & 1 \\
\hline S1-R7SO & 1 & $\mathrm{CA}$ & Soil & S1-R & 60 & 1.0 & 21 & 54 & 12 & 8 & 1 & 1 & 0 & 0 & 2 \\
\hline S1-A1SO & 1 & RA & Soil & S1-A & 5 & 1.0 & 14 & 60 & 10 & 7 & 6 & 3 & 0 & 0 & 0 \\
\hline \multirow[t]{4}{*}{ S1-T1SO } & 1 & FD & Soil & S1-T-UP & 5 & $<0.5$ & 17 & 47 & 16 & 9 & 0 & 0 & 7 & 0 & 4 \\
\hline & 1 & FD & Soil & S1-T-UP & 10 & $<0.5$ & 10 & 40 & 36 & 10 & 0 & 0 & 0 & 0 & 4 \\
\hline & 1 & FD & Soil & S1-T-UP & 15 & $<0.5$ & 12 & 68 & 13 & 6 & 0 & 0 & 0 & 0 & 1 \\
\hline & 1 & FD & Soil & S1-T-UP & 20 & $<0.5$ & 13 & 44 & 32 & 8 & 0 & 0 & 0 & 0 & 3 \\
\hline S1-T6SO & 1 & CRPM & Soil & S1-T-LOW & 40 & 0.5 & n.a. & n.a. & n.a. & n.a. & n.a. & n.a. & n.a. & n.a. & n.a. \\
\hline S1-T8SO & 1 & CRPM & Soil & S1-T-LOW & 60 & 0.5 & 26 & 50 & 7 & 9 & 1 & 0 & 5 & 1 & 2 \\
\hline S1-T10SO & 1 & CRPM & Soil & S1-T-LOW & 80 & 0.5 & 19 & 38 & 17 & 14 & 1 & 1 & 0 & 0 & 10 \\
\hline S2-SSNO1 & 2 & CRPM & Nodule & S2-S & 0 & 73.0 & n.a. & n.a. & n.a. & n.a. & n.a. & n.a. & n.a. & n.a. & n.a. \\
\hline S2-S1NO & 2 & CRPM & Nodule & S2-S & 5 & 78.5 & n.a. & n.a. & n.a. & n.a. & n.a. & n.a. & n.a. & n.a. & n.a. \\
\hline S2-S5NO & 2 & CRPM & Nodule & S2-S & 20 & 80.5 & n.a. & n.a. & n.a. & n.a. & n.a. & n.a. & n.a. & n.a. & n.a. \\
\hline S2-S20NO & 2 & CRPM & Nodule & S2-S & 60 & 76.5 & n.a. & n.a. & n.a. & n.a. & n.a. & n.a. & n.a. & n.a. & n.a. \\
\hline S2-S1SO & 2 & CRPM & Soil & S2-S & 5 & 6.0 & 33 & 48 & 4 & 4 & 5 & 0 & 0 & 0 & 4 \\
\hline S2-S5SO & 2 & CRPM & Soil & S2-S & 20 & 4.5 & 29 & 42 & 14 & 8 & 4 & 0 & 0 & 1 & 2 \\
\hline S2-S20SO & 2 & CRPM & Soil & S2-S & 60 & 4.0 & 37 & 22 & 16 & 3 & 5 & 0 & 0 & 0 & 16 \\
\hline S3-VSNO1 & 3 & CRPM & Nodule & S3-V & 0 & 81.5 & 39 & 9 & 0 & 0 & 46 & 0 & 0 & 0 & 5 \\
\hline S3-V1NO & 3 & CRPM & Nodule & S3-V & 5 & n.a & n.a. & n.a. & n.a. & n.a. & n.a. & n.a. & n.a. & n.a. & n.a. \\
\hline S3-V4NO & 3 & CRPM & Nodule & S3-V & 20 & n.a & n.a. & n.a. & n.a. & n.a. & n.a. & n.a. & n.a. & n.a. & n.a. \\
\hline S3-V8NO & 3 & CRPM & Nodule & S3-V & 60 & n.a & n.a. & n.a. & n.a. & n.a. & n.a. & n.a. & n.a. & n.a. & n.a. \\
\hline S3-V1SO & 3 & CRPM & Soil & S3-V & 5 & 2.0 & 40 & 34 & 8 & 4 & 1 & 0 & 2 & 0 & 12 \\
\hline S3-V4SO & 3 & CRPM & Soil & S3-V & 20 & 1.5 & 28 & 37 & 20 & 5 & 1 & 0 & 2 & 0 & 8 \\
\hline S3-V8SO & 3 & CRPM & Soil & S3-V & 60 & 1.5 & 38 & 34 & 11 & 3 & 1 & 0 & 2 & 0 & 11 \\
\hline GR & 1 & granite & Bedrock & & 0 & - & 6 & 21 & 27 & 42 & 0 & 0 & 0 & 0 & 4 \\
\hline RV & 3 & Green rock & Bedrock & & 0 & - & 34 & 9 & 3 & 16 & 0 & 0 & 0 & 0 & 37 \\
\hline Plg-granite & 1 & From granite & & & 0 & - & - & - & - & - & - & - & - & - & - \\
\hline Bte-granite & 1 & From granite & & & 0 & & & & & & & & & & \\
\hline PlgF8 & 1 & From F8SO & & S1-F & 60 & - & - & - & - & - & - & - & - & - & - \\
\hline PlgN8 & 1 & From N8SO & & S1-N & 60 & - & - & - & - & - & - & - & - & - & - \\
\hline Plg S20 & 2 & From S20SO & & S2-S & 60 & - & - & - & - & - & - & - & - & - & - \\
\hline PlgRV & 3 & From green & & & 0 & - & - & - & - & - & - & - & - & - & - \\
\hline E1 & 1 & Intermittent & eam & Water & - & - & - & - & - & - & - & - & - & - & - \\
\hline E2 & 1 & Intermittent & eam & Water & - & - & - & - & - & - & - & - & - & - & - \\
\hline E3 & 1 & Intermittent & eam & Water & - & - & - & - & - & - & - & - & - & - & - \\
\hline $\mathrm{E} 4$ & 1 & Intermittent & eam & Water & - & - & - & - & - & - & - & - & - & - & - \\
\hline E5 & 1 & Intermittent & eam & Water & - & - & - & - & - & - & - & - & - & - & - \\
\hline E6 & 1 & Kaliao afflue & & & - & - & - & - & - & - & - & - & - & - & - \\
\hline
\end{tabular}

An aliquot of each sample was used to measure total $\mathrm{Ca}, \mathrm{Sr}$, and $\mathrm{Nd}$ concentrations with an ICP-MS (Element XR) at Lausanne University, with an average uncertainty better than $5 \%$. For the soil residual phases, dissolutions were not totally complete. Thus, it was decided to analyse these samples in their solid form (residual phases after $2.2 \mathrm{M}$ acetic acid extraction). After $\mathrm{LiBO}_{2}$ fusion, these samples were analysed with a LA-ICP-MS for $\mathrm{Ca}, \mathrm{Sr}$, and Nd total contents. Triplicate analyses were performed on a nodule sample in order to control the reproducibility of the acid acetic extractions. Results gave a reproducibility standard deviation (RSD) of $0.9 \%$ for $\mathrm{Ca}, 0.6 \%$ for $\mathrm{Sr}$, and $0.002 \%$ for
${ }^{87} \mathrm{Sr} /{ }^{86} \mathrm{Sr}$ (Supplement materials). Ca, Sr, and $\mathrm{Nd}$ concentrations, as well as ${ }^{87} \mathrm{Sr} /{ }^{86} \mathrm{Sr}$ and $\varepsilon(\mathrm{Nd})$ are given in Table 2.

\section{Results}

\subsection{Carbonate content and mineralogy}

The carbonate content in the CRPM at sites 1 and 2 (S1-N, S1-F, S2S) varies between $1 \%$ and $6 \%$ in mass. Its mineralogical composition is mainly quartz, phyllosilicates (mainly smectites, but also kaolinite and 
Table 2

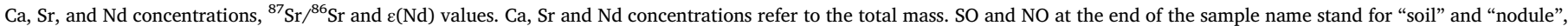
respectively. $\mathrm{Ca} / \mathrm{Sr}$ are expressed in molar ratios, whereas $\mathrm{Sr} / \mathrm{Nd}$ are mass ratios.

Acid acetic leached nodules and soils

\begin{tabular}{|c|c|c|c|c|c|c|}
\hline & Ca ppm & Sr ppm & $\mathrm{Ca} / \mathrm{Sr}$ molar & ${ }^{86} \mathrm{Sr} /{ }^{87} \mathrm{Sr}$ & & $2 \sigma$ \\
\hline S1-FSNO1 & 295,265 & 379.5 & 1704 & 0.71078 & \pm & 0.000005 \\
\hline S1-FSNO2 & 282,632 & 343.4 & 1803 & 0.71066 & \pm & 0.000007 \\
\hline S1-F1NO & 282,793 & 788.5 & 785 & 0.71084 & \pm & 0.000004 \\
\hline S1-F4NO & 304,320 & 504.9 & 1320 & 0.71080 & \pm & 0.000005 \\
\hline S1-F8NO & 254,257 & 474.3 & 1174 & 0.71070 & \pm & 0.000005 \\
\hline S1-F1SO & 15,029 & 71.3 & 461 & 0.71141 & \pm & 0.000004 \\
\hline S1-F4SO & 9671 & 62.4 & 339 & 0.71134 & \pm & 0.000004 \\
\hline S1-F8SO & 4376 & 41.0 & 234 & 0.71134 & \pm & 0.000007 \\
\hline S1-NSNO1 & 266,592 & 313.0 & 1866 & 0.71175 & \pm & 0.000007 \\
\hline S1-NSNO2 & 268,922 & 338.0 & 1743 & 0.71175 & \pm & 0.000006 \\
\hline S1-NSNO3 & 257,272 & 287.6 & 1959 & 0.71171 & \pm & 0.000006 \\
\hline S1-N1NO & 287,582 & 339.2 & 1857 & 0.71167 & \pm & 0.000002 \\
\hline S1-N4NO & 308,274 & 439.3 & 1537 & 0.71134 & \pm & 0.000002 \\
\hline S1-N8NO & 195,596 & 673.5 & 636 & 0.71135 & \pm & 0.000003 \\
\hline S1-N1SO & 6748 & 33.4 & 443 & 0.71165 & \pm & 0.000006 \\
\hline S1-N4SO & 7593 & 48.0 & 346 & 0.71149 & \pm & 0.000005 \\
\hline S1-N8SO & 3952 & 49.9 & 174 & 0.71146 & \pm & 0.000005 \\
\hline S1-R0SO & 1906 & 10.2 & 410 & 0.71196 & \pm & 0.000008 \\
\hline S1-R3SO & 1323 & 7.7 & 375 & 0.71179 & \pm & 0.000011 \\
\hline S1-R7SO & 1772 & 10.7 & 364 & 0.71161 & \pm & 0.000009 \\
\hline S1-A1SO & 3486 & 21.5 & 356 & 0.71155 & \pm & 0.000008 \\
\hline S1-T6SO & 852 & 9.8 & 190 & 0.71048 & \pm & 0.000012 \\
\hline S1-T8SO & 1585 & 19.2 & 180 & 0.71021 & \pm & 0.000009 \\
\hline S1-T10SO & 1388 & 16.8 & 181 & 0.71032 & \pm & 0.000008 \\
\hline S2-SSNO1 & 268,511 & 274.6 & 2141 & n.a & & \\
\hline S2-S1NO & 310,485 & 790.2 & 861 & 0.71224 & \pm & 0.000007 \\
\hline S2-S5NO & 303,727 & 570.0 & 1167 & 0.71244 & \pm & 0.000008 \\
\hline S2-S20NO & 290,065 & 432.1 & 1470 & 0.71288 & \pm & 0.000010 \\
\hline S2-S1SO & 19,785 & 114.0 & 380 & 0.71261 & \pm & 0.000009 \\
\hline S2-S5SO & 13,189 & 100.7 & 287 & 0.71271 & \pm & 0.000009 \\
\hline S2-S20SO & 13,275 & 90.6 & 321 & 0.71279 & \pm & 0.000008 \\
\hline S3-VSNO1 & 315,196 & 156.7 & 4406 & 0.70906 & \pm & 0.000003 \\
\hline S3-V1NO & 314,469 & 105.2 & 6549 & 0.70914 & \pm & 0.000012 \\
\hline S3-V4NO & 291,125 & 93.6 & 6815 & 0.70901 & \pm & 0.000005 \\
\hline S3-V8NO & 310,915 & 83.5 & 8153 & 0.70912 & \pm & 0.000006 \\
\hline S3-V1SO & 7138 & 18.6 & 841 & 0.70949 & \pm & 0.000010 \\
\hline S3-V4SO & 6597 & 18.6 & 776 & 0.70933 & \pm & 0.000007 \\
\hline S3-V8SO & 6573 & 19.5 & 736 & 0.70918 & \pm & 0.000009 \\
\hline
\end{tabular}

Residue nodules and soils

\begin{tabular}{|c|c|c|c|c|c|c|c|c|c|c|c|c|}
\hline & Ca ppm & Sr ppm & $\mathrm{Ca} / \mathrm{Sr}$ molar & ${ }^{86} \mathrm{Sr} /{ }^{87} \mathrm{Sr}$ & & $2 \sigma$ & Nd ppm & $\mathrm{Sr} / \mathrm{Nd}$ mass & $\varepsilon N d$ & ${ }^{143} \mathrm{Nd} /{ }^{144} \mathrm{Nd}$ & & $2 \sigma$ \\
\hline S1-FSNO1 & 9440 & 142.7 & 145 & 0.72089 & \pm & 0.000011 & 9.44 & 15.12 & -11.7 & 0.512038 & \pm & 0.000002 \\
\hline S1-FSNO2 & 2456 & 40.5 & 133 & 0.72072 & \pm & 0.000012 & 9.10 & 4.45 & -11.4 & 0.512055 & \pm & 0.000003 \\
\hline S1-F1NO & 2975 & 42.4 & 154 & 0.72024 & \pm & 0.000010 & 11.78 & 3.60 & -11.6 & 0.512045 & \pm & 0.000003 \\
\hline S1-F4NO & 1785 & 27.8 & 141 & 0.72038 & \pm & 0.000012 & 9.78 & 2.84 & -11.4 & 0.512052 & \pm & 0.000003 \\
\hline S1-F8NO & 4930 & 49.1 & 220 & 0.71901 & \pm & 0.000010 & 21.02 & 2.34 & -11.3 & 0.512061 & \pm & 0.000002 \\
\hline S1-F1SO & 5827 & 140.4 & 91 & 0.72120 & \pm & 0.000005 & 38.59 & 3.49 & -11.6 & 0.512042 & \pm & 0.000002 \\
\hline S1-F4SO & 5819 & 149.4 & 85 & 0.72059 & \pm & 0.000004 & 37.57 & 2.82 & -11.6 & 0.512045 & \pm & 0.000002 \\
\hline S1-F8SO & 5856 & 171.9 & 75 & 0.71936 & \pm & 0.000005 & 39.94 & 2.79 & -11.8 & 0.512031 & \pm & 0.000003 \\
\hline S1-NSNO1 & 2744 & 52.5 & 115 & 0.71913 & \pm & 0.000008 & 10.43 & 5.03 & -11.8 & 0.512034 & \pm & 0.000002 \\
\hline S1-NSNO2 & 2265 & 39.5 & 125 & 0.71927 & \pm & 0.000010 & 7.06 & 5.60 & -11.8 & 0.512034 & \pm & 0.000003 \\
\hline S1-NSNO3 & 2765 & 49.8 & 122 & 0.72028 & \pm & 0.000009 & 9.60 & 5.19 & -12.0 & 0.512021 & \pm & 0.000002 \\
\hline S1-N1NO & 3573 & 53.4 & 147 & 0.71937 & \pm & 0.000008 & 13.38 & 3.99 & -11.8 & 0.512035 & \pm & 0.000002 \\
\hline S1-N4NO & 1672 & 25.8 & 142 & 0.71917 & \pm & 0.000009 & 4.31 & 6.00 & -12.1 & 0.512020 & \pm & 0.000003 \\
\hline S1-N8NO & 3556 & 45.9 & 170 & 0.71712 & \pm & 0.000057 & 4.96 & 9.25 & -12.0 & 0.512021 & \pm & 0.000003 \\
\hline S1-N1SO & 6250 & 177.9 & 77 & 0.71964 & \pm & 0.000009 & 33.21 & 3.12 & -11.8 & 0.512035 & \pm & 0.000001 \\
\hline S1-N4SO & 6068 & 181.0 & 73 & 0.71961 & \pm & 0.000020 & 31.57 & 2.74 & n.a & n.a & & \\
\hline S1-N8SO & 6107 & 196.9 & 68 & 0.71921 & \pm & 0.000006 & 33.83 & 2.88 & -11.9 & 0.512030 & \pm & 0.000002 \\
\hline S1-R0SO & 4439 & 175.5 & 55 & 0.71977 & \pm & 0.000010 & 29.08 & 3.05 & -11.3 & 0.512061 & \pm & 0.000010 \\
\hline S1-R3SO & 3591 & 156.8 & 50 & 0.72068 & \pm & 0.000010 & 21.06 & 2.94 & -11.4 & 0.512052 & \pm & 0.000014 \\
\hline S1-R7SO & 4449 & 159.0 & 61 & 0.72084 & \pm & 0.000008 & 31.09 & 3.02 & -12.4 & 0.512000 & \pm & 0.000023 \\
\hline S1-A1SO & 5145 & 175.1 & 64 & 0.72001 & \pm & 0.000010 & 41.19 & 3.09 & -12.6 & 0.511992 & \pm & 0.000008 \\
\hline S1-T6SO & 5425 & 232.5 & 51 & 0.71784 & \pm & 0.000008 & 18.11 & 2.42 & -12.6 & 0.511991 & \pm & 0.000015 \\
\hline S1-T8SO & 6188 & 217.9 & 62 & 0.71673 & \pm & 0.000008 & 43.04 & 3.01 & -11.0 & 0.512075 & \pm & 0.000008 \\
\hline S1-T10SO & 7584 & 265.9 & 62 & 0.71439 & \pm & 0.000008 & 33.16 & 2.28 & -11.3 & 0.512059 & \pm & 0.000004 \\
\hline S2-SSNO1 & 2468 & 31.9 & 169 & 0.71948 & \pm & 0.000008 & 6.00 & 5.32 & -11.8 & 0.512032 & \pm & 0.000006 \\
\hline S2-S1NO & n.a. & n.a. & n.a. & 0.71817 & \pm & 0.000007 & n.a. & n.a. & -10.8 & 0.512085 & \pm & 0.000006 \\
\hline
\end{tabular}


Table 2 (continued)

Residue nodules and soils

\begin{tabular}{|c|c|c|c|c|c|c|c|c|c|c|c|c|}
\hline & Ca ppm & Sr ppm & $\mathrm{Ca} / \mathrm{Sr}$ molar & ${ }^{86} \mathrm{Sr} /{ }^{87} \mathrm{Sr}$ & & $2 \sigma$ & Nd ppm & $\mathrm{Sr} / \mathrm{Nd}$ mass & $\varepsilon N d$ & ${ }^{143} \mathrm{Nd} /{ }^{144} \mathrm{Nd}$ & & $2 \sigma$ \\
\hline S2-S5NO & n.a. & n.a. & n.a. & 0.71937 & \pm & 0.000008 & n.a. & n.a. & -11.8 & 0.512033 & \pm & 0.000007 \\
\hline S2-S20NO & n.a. & n.a. & n.a. & 0.72309 & \pm & 0.000010 & n.a. & n.a. & -11.0 & 0.512073 & \pm & 0.000006 \\
\hline S2-S1SO & 6870 & 161.4 & 93 & 0.71999 & \pm & 0.000010 & 36.45 & 2.91 & -10.3 & 0.512109 & \pm & 0.000012 \\
\hline S2-S5SO & 6686 & 166.4 & 88 & 0.71991 & \pm & 0.000009 & 37.79 & 2.92 & -9.9 & 0.512132 & \pm & 0.000013 \\
\hline S2-S20SO & 6145 & 147.4 & 91 & 0.72167 & \pm & 0.000008 & 30.64 & 2.99 & -12.2 & 0.512014 & \pm & 0.000011 \\
\hline S3-VSNO1 & 3911 & 29.4 & 292 & 0.71238 & \pm & 0.000011 & 6.04 & 4.87 & -8.1 & 0.512202 & \pm & 0.000007 \\
\hline S3-V1NO & 3354 & 19.4 & 379 & 0.71161 & \pm & 0.000007 & 7.18 & 2.70 & -8.0 & 0.512208 & \pm & 0.000014 \\
\hline S3-V4NO & 3996 & 29.6 & 295 & 0.71153 & \pm & 0.000011 & 5.51 & 5.38 & -6.8 & 0.512270 & \pm & 0.000009 \\
\hline S3-V8NO & 3554 & 26.5 & 294 & 0.71097 & \pm & 0.000002 & 5.29 & 5.01 & -8.2 & 0.512197 & \pm & 0.000014 \\
\hline S3-V1SO & 15,128 & 147.2 & 225 & 0.71225 & \pm & 0.000012 & 26.67 & 5.84 & -7.9 & 0.512212 & \pm & 0.000003 \\
\hline S3-V4SO & 15,733 & 152.6 & 226 & 0.71217 & \pm & 0.000022 & 26.76 & 4.52 & -6.8 & 0.512272 & \pm & 0.000002 \\
\hline S3-V8SO & 15,356 & 148.7 & 226 & 0.71139 & \pm & 0.000005 & 25.62 & 4.59 & -7.5 & 0.512236 & \pm & 0.000002 \\
\hline
\end{tabular}

Bulk

\begin{tabular}{|c|c|c|c|c|c|c|c|c|c|c|c|c|}
\hline & Ca ppm & $\mathrm{Sr} \mathrm{ppm}$ & $\mathrm{Ca} / \mathrm{Sr}$ molar & ${ }^{86} \mathrm{Sr} /{ }^{87} \mathrm{Sr}$ & & $2 \sigma$ & Nd ppm & $\mathrm{Sr} / \mathrm{Nd}$ mass & $\varepsilon \mathrm{Nd}$ & ${ }^{143} \mathrm{Nd} /{ }^{144} \mathrm{Nd}$ & & $2 \sigma$ \\
\hline S1-T1SO & 5245 & 207.6 & 55 & 0.71795 & \pm & 0.000009 & 23.63 & 2.824 & -13.9 & 0.511925 & \pm & 0.000002 \\
\hline S1-T2SO & 5100 & 205.0 & 54 & 0.71836 & \pm & 0.000005 & 20.20 & 2.977 & -16.3 & 0.511805 & \pm & 0.000002 \\
\hline S1-T3SO & 4937 & 192.3 & 56 & 0.71843 & \pm & 0.000005 & 22.78 & 2.824 & -13.3 & 0.511954 & \pm & 0.000003 \\
\hline S1-T4SO & 4700 & 185.9 & 55 & 0.71866 & \pm & 0.000005 & 20.25 & 3.482 & -12.6 & 0.511993 & \pm & 0.000003 \\
\hline GR & 8382 & 248.7 & 74 & 0.72340 & \pm & 0.000007 & 28.90 & 8.606 & -9.6 & 0.512145 & \pm & 0.000003 \\
\hline RV & 79,122 & 274.8 & 631 & 0.70349 & \pm & 0.000004 & 6.71 & 40.959 & 4.5 & 0.512870 & \pm & 0.000005 \\
\hline Bte-Granite & n.a. & 168.0 & - & 0.73912 & \pm & 0.000008 & n.a. & n.a. & n.a. & n.a. & & \\
\hline Plg-granite & 6915 & 288.4 & 53 & 0.71180 & \pm & 0.000004 & n.a. & n.a. & n.a. & n.a. & & \\
\hline PlgF8 & 12,475 & 369.3 & 74 & 0.70688 & \pm & 0.000020 & n.a. & n.a. & n.a. & n.a. & & \\
\hline PlgN8 & 10,186 & 251.2 & 89 & 0.70859 & \pm & 0.000020 & n.a. & n.a. & n.a. & n.a. & & \\
\hline Plg S20 & 9806 & 250.0 & 86 & 0.70982 & \pm & 0.000020 & n.a. & n.a. & n.a. & n.a. & & \\
\hline PlgRV & 30,967 & 327.9 & 207 & 0.70589 & \pm & 0.000020 & n.a. & n.a. & n.a. & n.a. & & \\
\hline E1 & 6.785 & 0.050 & 297 & 0.71120 & \pm & 0.000018 & n.a. & n.a. & n.a. & n.a. & & \\
\hline E2 & 2.947 & 0.024 & 269 & 0.71116 & \pm & 0.000018 & n.a. & n.a. & n.a. & n.a. & & \\
\hline E3 & 4.850 & 0.039 & 272 & 0.71099 & \pm & 0.000018 & n.a. & n.a. & n.a. & n.a. & & \\
\hline E4 & 25.193 & 0.186 & 297 & 0.71091 & \pm & 0.000018 & n.a. & n.a. & n.a. & n.a. & & \\
\hline E5 & 39.670 & 0.295 & 294 & 0.71105 & \pm & 0.000018 & n.a. & n.a. & n.a. & n.a. & & \\
\hline E6 & 7.578 & 0.066 & 251 & 0.71186 & \pm & 0.000018 & n.a. & n.a. & n.a. & n.a. & & \\
\hline
\end{tabular}

illite in smaller amounts), feldspars, and plagioclases, in descending order (Table 1). The carbonate contents in S3-V samples are slightly higher $(5.5 \%$ to $9 \%)$ and the abundance of minerals is different compared to the other profiles (plagioclases, feldspar, quartz, phyllosilicates as smectites, and epidote, by descending order, respectively). In carbonate nodules, the carbonate content varies from $70 \%$ to $81.5 \%$. The most abundant mineral is calcite, while the residual silicate fraction is similar to the composition of the bulk soil. The S1-R carbonate content is $<1 \%$. The mineralogical composition is mainly quartz, feldspar, phyllosilicates, and plagioclases, in descending order. S1-A sample has a carbonate content of $1 \%$ and a mineralogy similar to S1-R. The S1-T carbonate content is $0.5 \%$. The mineralogical composition is mainly quartz, phyllosilicates, feldspars, and plagioclases, in descending order. Finally, the granite of the area has a porphyritic texture and is composed of plagioclases, feldspars, quartz, and biotites. No apatite was observed. Maroua greenstone includes plagioclases, feldspar, quartz, and epidotes.

\subsection{Sr and Nd isotopic compositions}

\subsubsection{Sr isotopic compositions of the carbonate phases}

The $\mathrm{Sr}$ isotopic composition of the various carbonate phases (extracted with the 2.2. M acetic acid from the soil and nodules) is quite homogeneous, ranging from 0.7090 to 0.7129 . However, each site displays a specific range of ${ }^{87} \mathrm{Sr} /{ }^{86} \mathrm{Sr}$ values (Fig. 4A). At site 1 , ${ }^{87} \mathrm{Sr} /{ }^{86} \mathrm{Sr}$ ratios of the carbonate phases are relatively homogenous in S1-F and S1-N profiles $\left({ }^{87} \mathrm{Sr} /{ }^{86} \mathrm{Sr}=0.7113 \pm 0.0004, \mathrm{n}=17,1 \sigma\right)$. ${ }^{87} \mathrm{Sr} /{ }^{86} \mathrm{Sr}$ ratios of S1-T are well centred around $0.7103 \pm 0.0001$
( $\mathrm{n}=3,1 \sigma$ ). The carbonate phases at profile S2-S have the most radiogenic signature $(0.7126 \pm 0.0002, \mathrm{n}=6,1 \sigma)$, while the less radiogenic signature $(0.7092 \pm 2, \mathrm{n}=7,1 \sigma \mathrm{Fig}$. 4B) is observed in profile S3-V. The soil carbonate displays ${ }^{87} \mathrm{Sr} /{ }^{86} \mathrm{Sr}$ signatures similar to the nodule carbonate, with the exception of the profile S1-F, for which soil carbonate has a slightly more radiogenic composition (Fig. 5A). It is noteworthy noting that $\mathrm{Ca} / \mathrm{Sr}$ ratios are systematically lower in the soil carbonate than in nodule carbonate (Fig. 4). Samples from surface waters have $\mathrm{Sr}$ isotopic signatures $(0.7112 \pm 3, \mathrm{n}=6)$ close to those of soil carbonate and nodules at site 1 (Fig. 4).

\subsubsection{Sr and Nd isotopic compositions of residual phases and rocks}

The ${ }^{87} \mathrm{Sr} /{ }^{86} \mathrm{Sr}$ ratios obtained in residual phases (residue from $2.2 \mathrm{M}$ acetic acid extraction on soil and nodules) are significantly more radiogenic and more heterogeneous than in the carbonate phases (Fig. 4A). A good correspondence is found between the ${ }^{87} \mathrm{Sr} /{ }^{86} \mathrm{Sr}$ ratios of the residue from soils and nodules (Fig. 5C). At site $1,{ }^{87} \mathrm{Sr} /{ }^{86} \mathrm{Sr}$ ratios of residual phases range from 0.7192 to 0.7212 , except for the S1-T and one nodule residue from profile S1-N (sample S1-N8-NO), which display lower ratios. Ratios in profile S2-S are more heterogeneous and can reach ${ }^{87} \mathrm{Sr} /{ }^{86} \mathrm{Sr}=0.7231$. In profile $\mathrm{S} 3-\mathrm{V},{ }^{87} \mathrm{Sr} /{ }^{86} \mathrm{Sr}$ ratios of the residual phases are markedly lower than those in sites 1 and 2, ranging from 0.7110 to 0.7124 . $\mathrm{Ca} / \mathrm{Sr}$ ratios are in the same range in both soil and nodule residues.

The granite sample has a ${ }^{87} \mathrm{Sr} /{ }^{86} \mathrm{Sr}$ value of 0.7234 and the Maroua greenstone of 0.7035 . Biotite extracted from the granite has the highest ${ }^{87} \mathrm{Sr} /{ }^{86} \mathrm{Sr}$ ratio $(0.7391) .{ }^{87} \mathrm{Sr} /{ }^{86} \mathrm{Sr}$ values of plagioclases range from 0.7059 to 0.7118 . Moreover, a significant similarity is observed 

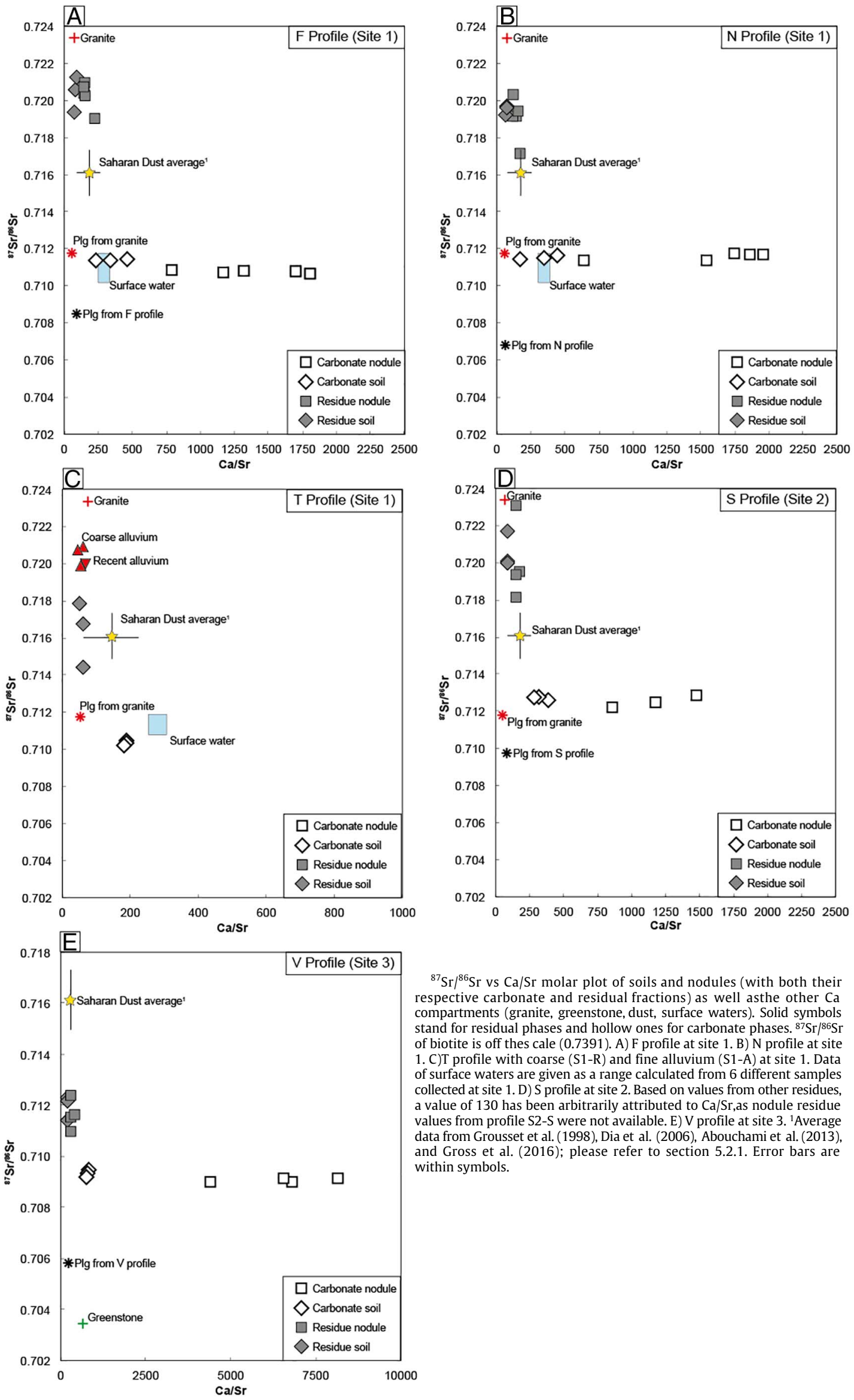

${ }^{87} \mathrm{Sr} /{ }^{86} \mathrm{Sr}$ vs $\mathrm{Ca} / \mathrm{Sr}$ molar plot of soils and nodules (with both their respective carbonate and residual fractions) as well asthe other $\mathrm{Ca}$ compartments (granite, greenstone, dust, surface waters). Solid symbols stand for residual phases and hollow ones for carbonate phases. ${ }^{87} \mathrm{Sr} /{ }^{86} \mathrm{Sr}$ of biotite is off thes cale (0.7391). A) F profile at site 1 . B) N profile at site 1. C)T profile with coarse (S1-R) and fine alluvium (S1-A) at site 1. Data of surface waters are given as a range calculated from 6 different samples collected at site 1.D) S profile at site 2 . Based on values from other residues, a value of 130 has been arbitrarily attributed to $\mathrm{Ca} / \mathrm{Sr}$,as nodule residue values from profile S2-S were not available. E) V profile at site 3. ${ }^{1}$ Average data from Grousset et al. (1998), Dia et al. (2006), Abouchami et al. (2013), and Gross et al. (2016); please refer to section 5.2.1. Error bars are within symbols. 


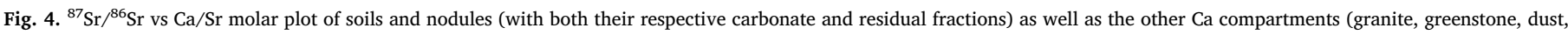

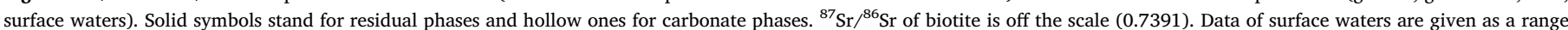

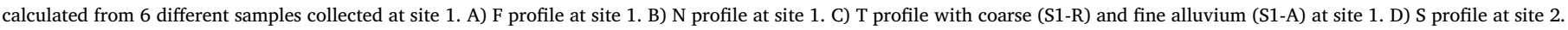

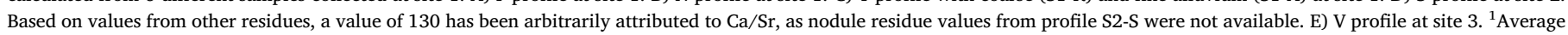
data from Grousset et al. (1998), Dia et al. (2006), Abouchami et al. (2013), and Gross et al. (2016); please refer to Section 5.2.1. Error bars are within symbols.

between ${ }^{87} \mathrm{Sr} /{ }^{86} \mathrm{Sr}$ ratios averaged per profile for carbonate nodules and plagioclases extracted from soils (S1-F, S1-N, S2-S) and greenstone (Fig. 5B).

Isotopic compositions of $\mathrm{Nd}$ display a fairly narrow range of values for the residual phases (either from soil or nodule), except for profile S3-V (Fig. 6). Granite $\varepsilon(\mathrm{Nd})$ is distinct from the other samples. All $\mathrm{Nd}$ isotopic compositions of samples at site 3 are more radiogenic than at sites 1 and 2. $\varepsilon(\mathrm{Nd})$ of the residual phases from profile S3-V range from -8.24 to -6.76 and the $\varepsilon(\mathrm{Nd})$ of Maroua green rock is 4.25 .

\section{Discussion}

\subsection{The local bedrock is a calcium source}

\subsubsection{A significant contribution of plagioclase to Ca supply}

$\mathrm{Sr}$ isotopic compositions of the greenstone and the granite are clearly distinct, with a more radiogenic signature for the granite as expected, due to biotite mineral occurrence. The ${ }^{87} \mathrm{Sr} /{ }^{86} \mathrm{Sr}$ ratio of the granite plots consistently between values of plagioclase and biotite. Differences in $\mathrm{Sr}$ isotopic compositions between plagioclases extracted from granite and from soil are also observed (Fig. 4A). Such variations have already been reported in the literature and can be attributed to the occurrence of clays in mineral micro-cracks (Clauer, 1981). Though, another likely explanation for the Northern Cameroon case is that the plagioclases from the outcropping granitic inselberg are not representative of the plagioclases collected from the soils. Indeed, inselbergs outcrop in the landscape because they are more resistant to weathering (due to their mineralogy/texture/structure) than the other surrounding rocks.

Carbonate from nodules and soils have the same ${ }^{87} \mathrm{Sr} /{ }^{86} \mathrm{Sr}$ ratio, emphasizing that they share the same Ca sources. However, at site 1, surface waters have a $\mathrm{Sr}$ isotopic composition close to that of carbonate (Fig. 4). This very likely indicates that the alkaline-earth composition of water in this watershed is controlled by the dissolution of the inherited carbonate. Carbonate phases have a distinct and specific ${ }^{87} \mathrm{Sr} /{ }^{86} \mathrm{Sr}$ signature at each site (Fig. 5A). In addition, ${ }^{87} \mathrm{Sr} /{ }^{86} \mathrm{Sr}$ ratios of the carbonate phases are lower in the greenstone area (site 3), compared to the granitic bedrock (site 1 and 2), illustrating the signature of the bedrocks. These two observations point at least to a partial local origin of $\mathrm{Ca}$ to explain the differences in the ${ }^{87} \mathrm{Sr} /{ }^{86} \mathrm{Sr}$ ratios observed in one hand between the different granitic sites and one the other hand between granitic and greenstone areas.

A co-variation is observed between $\mathrm{Sr}$ isotopic compositions of carbonate nodules and plagioclases extracted from soil. However, $\mathrm{Sr}$ isotopic compositions of carbonate nodules is slightly more radiogenic compared to plagioclases (Fig. 5B). This shift is relatively constant $(0.0032 \pm 0.0004 \mathrm{ppm})$ and the slope of the line joining the different profiles is close to 1 . Therefore, this co-variation of plagioclases and carbonate nodules suggests that plagioclases contributed to the $\mathrm{Ca}$ supply. Nevertheless, the shift between the ${ }^{87} \mathrm{Sr} /{ }^{86} \mathrm{Sr}$ ratios of carbonate nodules and plagioclases indicates that another source, more radiogenic, must be involved. This more radiogenic source may be either autochtonous or allochtonous. Consequently, in the next sections, the possible role of the other sediments present in the landscape, and a potential contribution of dust inputs in explaining the more radiogenic signature are explored.

\subsubsection{Identification of other potential calcium sources}

In the granite area (sites 1 and 2), the coarse alluvium is occasionally observed. It partly originated from granite weathering and was reworked during different sedimentary cycles (Hervieu, 1967; Morin, 2000; Diaz et al., 2016a). This coarse alluvium has a Sr isotopic composition slightly less radiogenic than the granite (Fig. 4C). This less radiogenic composition is probably due to the disappearance of biotite in this sediment, a highly weatherable mineral with a high radiogenic $\mathrm{Sr}$ isotopic signature. The coarse alluvium is only observed in the S1-R profile where it is interfingered with the CRPM as described by Morin (2000). It does not occur everywhere and has not been observed close to S1-F, S2-S or S3-V profiles. Consequently, a widespread contribution from the coarse alluvium (S1-R) to Sr, and hence $\mathrm{Ca}$, supply is unlikely. Logically, the recent alluvium (S1-A) has a Sr isotopic composition close to the CRPM, which supports field observations showing that it is mainly composed of reworked CRPM.

The CRPM is the soil parent material and the carbonate nodules precipitated within (Diaz et al., 2016a). This is supported by the similarity of the $\mathrm{Sr}$ isotopic compositions of the residue from the soils with the one from the nodules (Fig. 5C). Therefore, the CRPM undoubtedly plays a significant role in nodule formation, and other mineral fractions of the CRPM may provide a potential reservoir for $\mathrm{Sr}$ and $\mathrm{Ca}$. Apart from plagioclases, which have contributed to the $\mathrm{Ca}$ supply, the CRPM contains several other minerals originating from the weathering of the granititic bedrock, such as quartz, feldpar, and some biotite. The biotite sample extracted from the granite has a high $\mathrm{Sr}$ isotopic ratio $\left({ }^{86} \mathrm{Sr} /{ }^{87} \mathrm{Sr}=0.7391\right)$. A contribution of this phase, even small, might explain the shift between the Sr isotopic compositions of carbonate nodules and plagioclases (Fig. 5B).

Regarding the greenstone area, things are slightly different. The $\mathrm{Sr}$ isotopic signature of the greenstone is less radiogenic than that of CRPM and there is no radiogenic mineral originating from the greenstone that could contribute to an increase in the ${ }^{86} \mathrm{Sr} /{ }^{87} \mathrm{Sr}$ ratios of carbonate nodules and CRPM. Nonetheless, large amounts of granite outcrop upstream of the greenstone area and we cannot rules out that some granite-derived material, including biotites, may have been transported downstream and mixed with the CRPM located in the greenstone area. Consequently, the biotites could have contributed to supply some $\mathrm{Sr}$ to the the CRPM and thus to carbonate nodules, even in the greenstone area. Although this hypothesis is reasonable, some other allochtonous sources can contribute to a more radiogenic ${ }^{86} \mathrm{Sr} /{ }^{87} \mathrm{Sr}$ signature.

\subsubsection{Contributions from allochthonous sources}

Atmospheric inputs, as either rainwater or aerosols, have been identified by many authors as an important allochthonous source of $\mathrm{Ca}$ in various environments (e.g. Quade et al., 1995; Chiquet et al., 1999; Capo and Chadwick, 1999). In this study, atmospheric inputs could not be sampled. It is important to note that only long-term monitoring would have been appropriate to provide a representative and robust assessment of their chemical and isotopic signatures and contributions (see e.g. Skonieczny et al., 2011 for dust and Dupré et al., 1994 for rainwater). Such a thorough investigation would have required unwieldy and complicated logistic means that, for security reasons, were impossible to set up. Moreover, there is no available study reporting $\mathrm{Sr}$ isotopic composition of rainwater in the Far North region of Cameroon. $\mathrm{Sr}^{2+}$, as a dissolved cation in the rainwater, has two potential origins: the seawater, when close to the coastline; and aeolian particles, in the hinterland (Negrel et al., 1993; Derry and Chadwick, 2007; Négrel et al., 2007; Pearce et al., 2015). The study sites are located about $1000 \mathrm{~km}$ away from the coastline. At this distance, there is neither $\mathrm{Sr}$ nor $\mathrm{Ca}$ in rainwater that can reasonably originate from seawater; consequently, dust particles are favoured. This conclusion is also based on patterns observed in several studies, showing that the $\mathrm{Sr}$ contribu- 

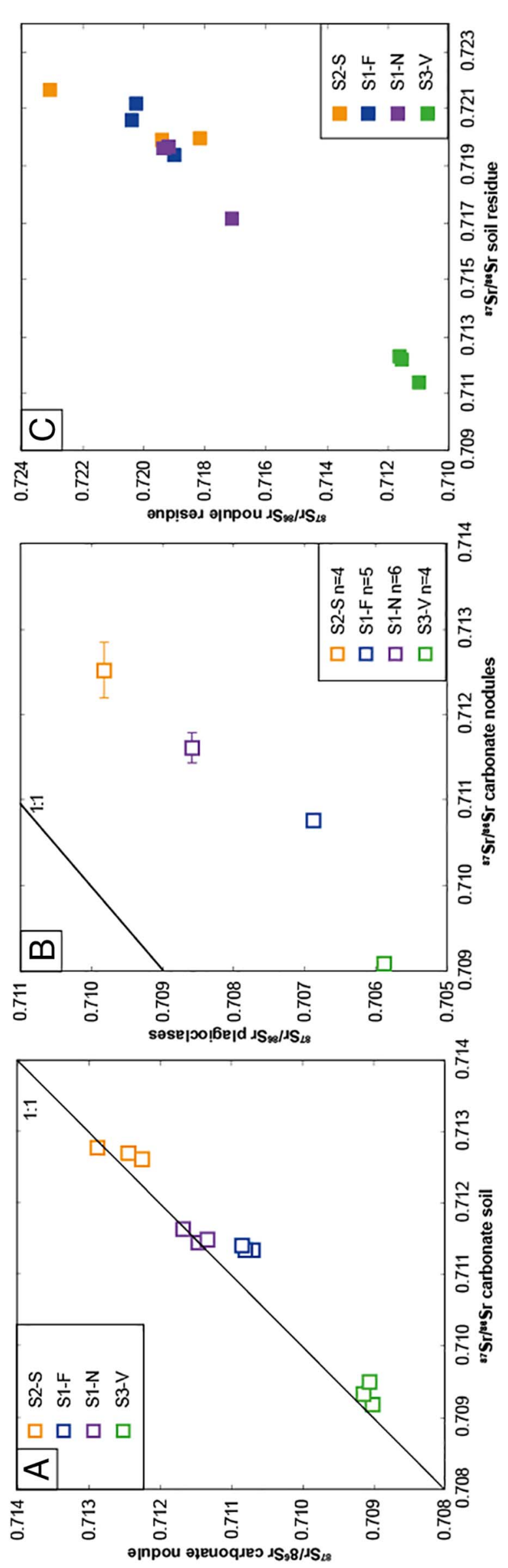

tion of seawater to secondary carbonate decreases with distance to the coastline (Quade et al., 1995; Rech et al., 2003).

Regarding dust, the Far North Region of Cameroon is situated downstream of sand seas and along a discontinuous desert loess belt extending from southern Senegal to northern Cameroon (Crouvi et al., 2010). In the study area, important dust deposits (the Doyang formation) were reported in the south bank of the Mayo Tsanaga (Mpeck, 1994; Lamotte et al., 1997). At the studied sites, grain-size distributions and micromorphological observations of the CRPM highlight the presence of well-sorted and smooth quartz grains, typical of aeolian deposits, supporting a possible contribution of aeolian sediments (Diaz et al., 2016a). Consequently, a contribution of dust to the CRPM, and thus potentially to the carbonate nodules, is likely. Therefore, it is necessary to estimate the $\mathrm{Sr}$ and $\mathrm{Nd}$ isotopic compositions of the Saharan dust in the Far North Region of Cameroon.

\subsection{The origin of the CRPM and the contribution of the Saharan dust}

\subsubsection{The isotopic signature of Saharan dust}

The study sites are located along the Harmattan wind pathway, loaded with Saharan dust (Kalu, 1979; Schwanghart and Schütt, 2008) originating mainly from the Bodélé Depression (Engelstaedter et al., 2006). Many studies have attempted to characterize the Sr and Nd isotopic compositions of the Saharan dust (e.g. Grousset et al., 1998; Skonieczny et al., 2011), and more specifically, of the Bodélé Depression source (Abouchami et al., 2013). The range reported in the literature is quite large (Fig. 6) and $\mathrm{Sr}$ and Nd signatures measured in aerosols and dust deposits may show contrasted signatures, regarding their departure dust sources (Abouchami et al., 2013). To approximate a representative signature of the regional dust, we only considered aerosols and dust deposits, and selected data from specific regions expected to be dust sources for the study area as it is located along specific wind trajectories: i) the Harmattan deposits in Faya Largeau and Mao in Chad, as well as in N'guigmi and Niamey, Niger (Abouchami et al., 2013); ii) dust deposits in Niamey (Grousset et al., 1998); iii) two soil samples composed mainly of dust from the Bodélé Depression (Gross et al., 2016); and finally, iv) a calculated value for the aeolian end-member that contributed to soils recovered from the Mount Cameroon (Dia et al., 2006). The calculated average value is $0.716 \pm 0.001(\mathrm{n}=8)$ and $-11.5 \pm 1.0(\mathrm{n}=6)$ for ${ }^{87} \mathrm{Sr} /{ }^{86} \mathrm{Sr}$ and $\varepsilon(\mathrm{Nd})$, respectively, and the $\mathrm{Ca} / \mathrm{Sr}$ ratio and the $\mathrm{Sr} / \mathrm{Nd}$ ratio are $179 \pm 81(\mathrm{n}=4)$ and $3.8 \pm 1.6(\mathrm{n}=6)$, respectively. The ${ }^{86} \mathrm{Sr} /{ }^{87} \mathrm{Sr}$ ratio estimated for the Saharan dust is therefore more radiogenic than those from the various carbonate phases. Consequently, Saharan dust constitutes a pertinent source that could have contributed to $\mathrm{Sr}$ and $\mathrm{Ca}$ supplies in carbonate nodules. Moreover, the $\mathrm{Sr}$ and $\mathrm{Nd}$ isotopic compositions of the Saharan dust are in the same range than the ones of CRPM (Fig. 6). At this stage, the composition of the CRPM and its origin appears as a key point, as it likely contains the potential Ca sources of the carbonate nodules.

\subsubsection{The mixed composition of CRPM}

${ }^{87} \mathrm{Sr} /{ }^{86} \mathrm{Sr}$ and and $\varepsilon(\mathrm{Nd})$ values of the CRPM (residue from soils and nodules) in the granitic area (site 1 and 2) are distributed between the values of granite and the Saharan dust average (Fig. 6). In the greenstone area (site 3), the Sr isotopic composition of CRPM is substantially more radiogenic than the one the greenstone (Fig. 4E). Moreover, $\varepsilon(\mathrm{Nd})$ values of the CRPM on site 3 and greenstone are clearly distinct (Fig. 7), supporting the fact that it cannot be only a weathering product of the greenstone. Another source, more radiogenic, is needed to explain the $\mathrm{Sr}$ and Nd isotopic signature of the CRPM on site 3. This radiogenic source could be the Saharan dust or/and minerals from the granite located upstream. Consequently, it is likely that the CRPM is composed of a mixture of three components: granite, greenstone, and the Saharan dust.

Regarding the two bedrocks, only one sample was available, 


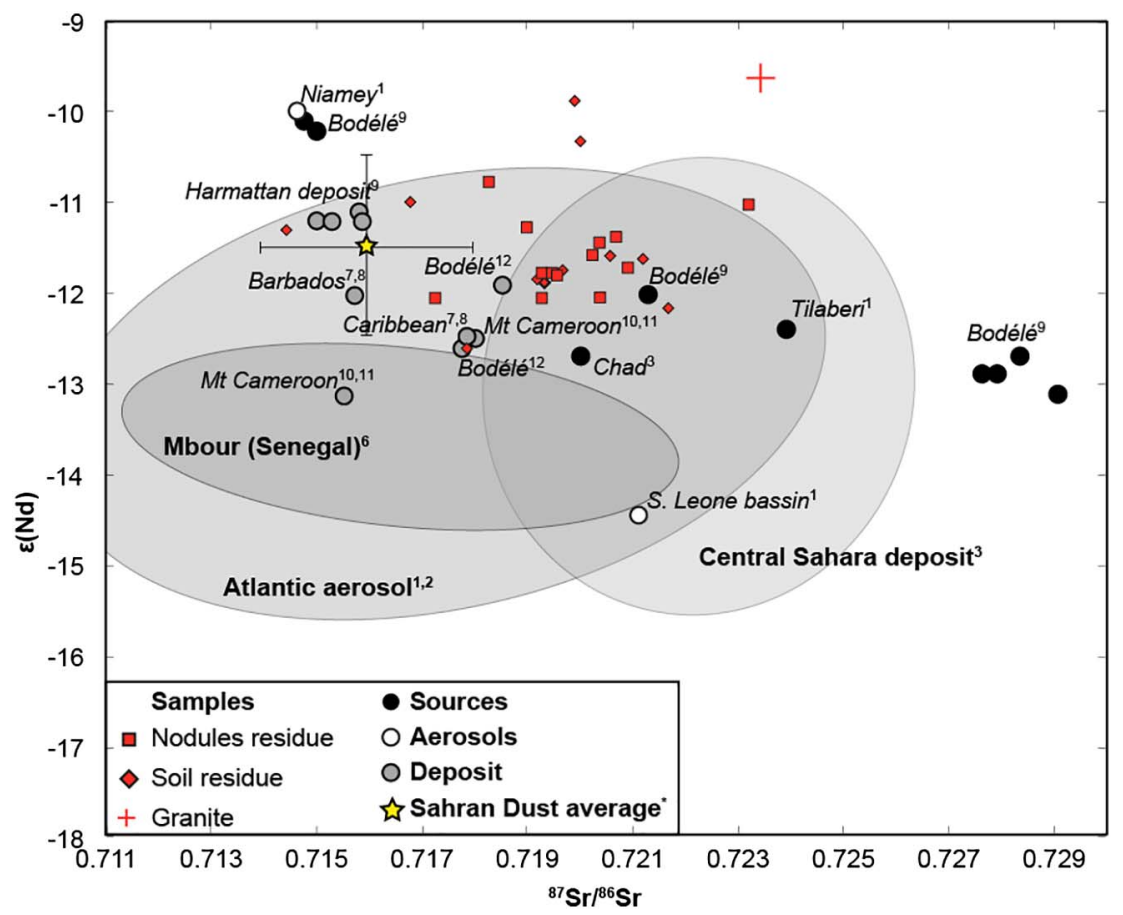

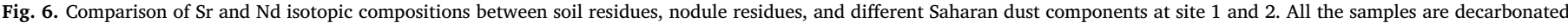

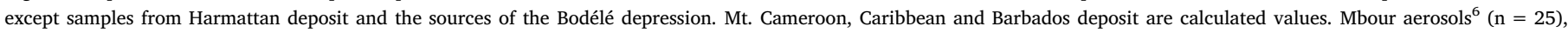

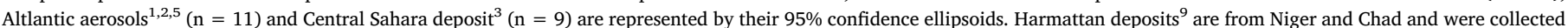

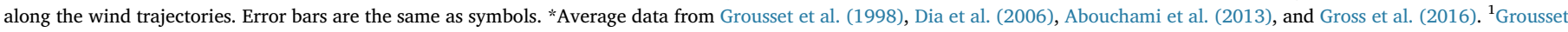

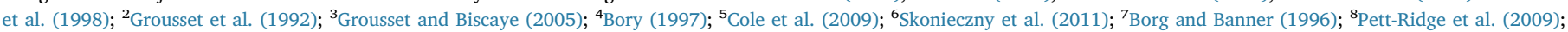
${ }^{9}$ Abouchami et al. (2013); ${ }^{10}$ Pelt et al. (2013); ${ }^{11}$ Dia et al. (2006); ${ }^{12}$ Gross et al. (2016).

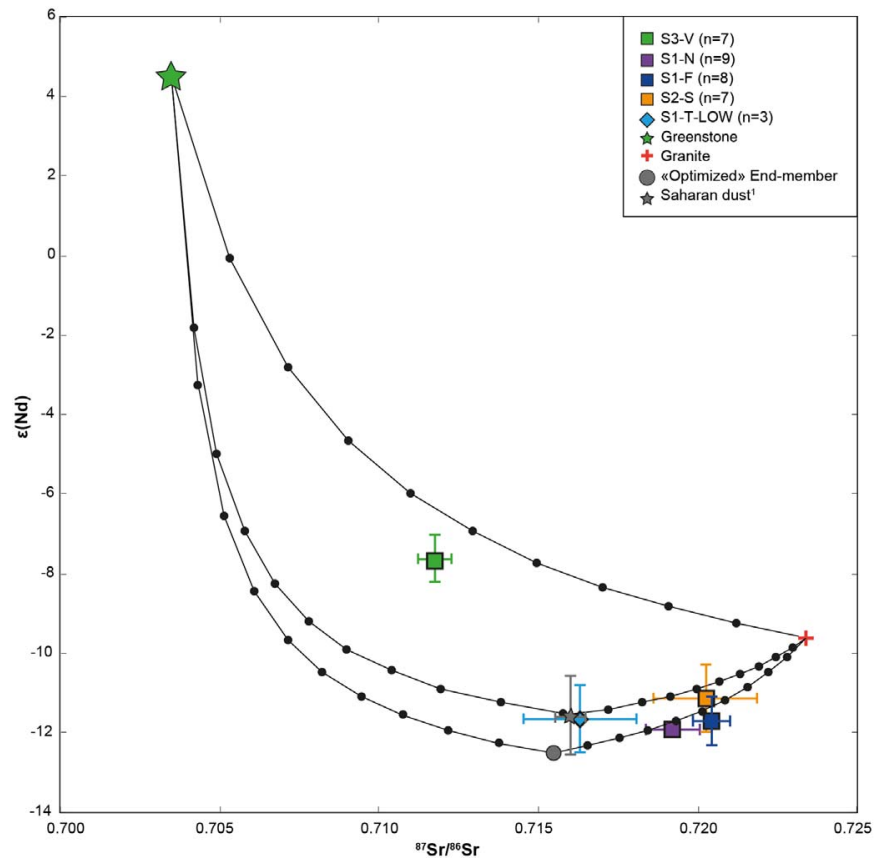

Fig. 7. ${ }^{87} \mathrm{Sr} /{ }^{86} \mathrm{Sr}$ ratios and $\varepsilon(\mathrm{Nd})$ of residue in the three studied sites. S1-F is in dark blue, $\mathrm{S} 1-\mathrm{N}$ in purple, S1-T in cyan, S2-S in orange, and S3-V in green. Data are averaged for soils and nodule residues. The black lines draw the hyperbolas between the "optimized" end-member/Saharan dust and the two bedrocks. ${ }^{1}$ Grousset et al. (1998), Dia et al. (2006), Abouchami et al. (2013) and Gross et al. (2016). Where not shown, error bars are the same as symbols. (For interpretation of the references to color in this figure legend, the reader is referred to the web version of this article.)

precluding any estimations of the variability of their isotopic signatures. Three mixing hyperbola have been plotted, involving these three end-members, i.e. granite, greenstone, and the Saharan dust (Fig. 7 and
Table 3). The first hyperbola connects the granitic bedrock and the Saharan dust. The second hyperbola joins the greenstone and the Saharan dust. A third hyperbola links greenstone and granite. However, the granite-dust hyperbola does not perfectly intersect the CRPM samples. In order to correct this, the value of the Saharan dust can be slightly changed by calculating an "optimized" end-member (Table 3; Dia et al., 2006) to better fit the samples (Fig. 7). Sr and Nd isotopic signatures of the "optimized" end-member are close to the estimated value of the Saharan dust. Regarding the S3-V profile, it does not fall on the plot of greenstone-granite hyperbola, as it is shifted towards the direction of greenstone-dust hyperbola. Thus, the CRPM at the greenstone area seems to be a mixture of the three end-members: two main contributions from the greenstone and the granite, and a third minor contribution from the Saharan dust. The respective contributions of Saharan dust and the local bedrock are not similar in each CRPM sample. In granite-dust hyperbola, S1-T includes $80 \pm 15 \%$ of the "optimized" Saharan dust, followed by S1-N (60\% $\pm 15 \%)$, S1-F, and S2-S $(40 \% \pm 15 \%)$. This is probably due to the geomorphological and stratigraphical positions of S1-T. Indeed, this profile is buried under a recent fersiallitic pediment. It does not result from erosion and is therefore different from sampled mima-like mounds (S1-F and S2-S). It has probably been less reworked/mixed with local granitic arena.

Taking into account the isotopic evidence exposed above and the geological and geomorphological data reported by Diaz et al. (2016a), the CRPM clearly appears as a mixture of Saharan dust and products from the weathered local bedrock. This reworking and mixture of dust and local weathered bedrock is common and has already been described in Mt. Cameroon (Dia et al., 2006). Furthermore, the determination of the various constituents forming the CRPM sheds light on the potential $\mathrm{Ca}$ sources of carbonate nodules. Indeed, aside from plagioclases from the local bedrock (granite at sites 1 and 2, and greenstone at site 3), the CRPM also contains Saharan dust and radiogenic minerals (biotite) from the granite at the three study sites. Consequently, Saharan dust and biotite must be considered as potential 
Table 3

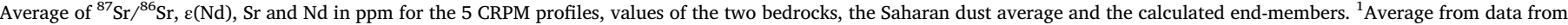
Grousset et al. (1998), Dia et al. (2006), Abouchami et al. (2013) and Gross et al. (2016).

\begin{tabular}{|c|c|c|c|c|c|c|c|c|c|c|c|c|c|}
\hline & $\mathrm{n}$ & ${ }^{87} \mathrm{Sr} /{ }^{86} \mathrm{Sr}$ & & $2 \sigma$ & $\varepsilon N d$ & & $2 \sigma$ & Sr ppm & & $2 \sigma$ & Nd ppm & & $2 \sigma$ \\
\hline Average S1-F & 8 & 0.72030 & \pm & 0.000754 & -11.5 & \pm & 0.2 & - & \pm & & - & \pm & \\
\hline Average S1-N & 9 & 0.71920 & \pm & 0.000860 & -11.9 & \pm & 0.1 & - & \pm & & - & \pm & \\
\hline Average S2-S & 3 & 0.72024 & \pm & 0.001631 & -11.1 & \pm & 0.9 & - & \pm & & - & \pm & \\
\hline Average S1-T-LOW & 3 & 0.71632 & \pm & 0.001764 & -11.6 & \pm & 0.9 & - & \pm & & - & \pm & \\
\hline Average S3-V & 7 & 0.71176 & \pm & 0.000484 & -7.6 & \pm & 0.6 & - & \pm & & - & \pm & \\
\hline Granite & 1 & 0.72340 & & - & -9.6 & & - & 249 & & - & 28 & & - \\
\hline Greenstone & 1 & 0.70348 & & - & 4.5 & & - & 275 & & - & 7 & & - \\
\hline Saharan dust average ${ }^{1}$ & 8 & 0.71607 & \pm & 0.000260 & -11.5 & \pm & 1.0 & 144 & \pm & 74 & 40 & \pm & 21 \\
\hline Optimized End-Member & & 0.71550 & & - & -12.5 & & - & 180 & & - & 50 & & - \\
\hline
\end{tabular}

Sr sources, and thus for Ca as well.

\subsection{Calculations of the respective contributions of Ca sources and implications}

\subsubsection{Calculations of respective contributions}

One of the Ca sources for carbonate nodules in the Far North Region of Cameroon has been demonstrated to be plagioclase from the local bedrock (Fig. 5B). However, a second source, characterized by a more radiogenic signature, must be considered. Two more radiogenic sources have been identified: Saharan dust and biotite from granite, but it remains challenging to differentiate which of them has the most influence at this stage. The Ca content of biotite has not been measured in this study, but it is generally very low. Nevertheless, even if biotite did not provide any Ca to the carbonate nodules, it can contribute to radiogenic $\mathrm{Sr}$, increasing the ${ }^{87} \mathrm{Sr} /{ }^{86} \mathrm{Sr}$ ratio of carbonate nodules. Consequently, three sources (i.e plagioclase, biotite, and Saharan dust) have to be considered in the calculation of their respective $\mathrm{Ca}$ or $\mathrm{Sr}$ contributions to carbonate nodules. Unfortunately, a calculation with three end-members is difficult and cannot be performed with the available data ( $\mathrm{Sr} / \mathrm{Ca}$ ratio of the biotite is unknown). Therefore, the contributions of two different mixtures are calculated: i) plagioclase and biotite on one hand, but only for the $\mathrm{Sr}$ contribution; ii) plagioclase and Saharan dust on the other hand, to evaluate their respective $\mathrm{Ca}$ contributions. Respective $\mathrm{Ca}$ and $\mathrm{Sr}$ contributions can be calculated using equations proposed by Capo et al. (1998):

$$
\begin{aligned}
& \% \mathrm{Ca} \text { EM2 } \\
& \left.\left.=\frac{\left(\left({ }^{87} \mathrm{Sr}\right.\right.}{{ }^{86} \mathrm{Sr}}\right)_{\mathrm{MIX}}-\left(\frac{87 \mathrm{Sr}}{{ }^{86 \mathrm{Sr}}}\right)_{\mathrm{EM} 1}\right) * \mathrm{~K}_{\mathrm{EM} 1}
\end{aligned}
$$

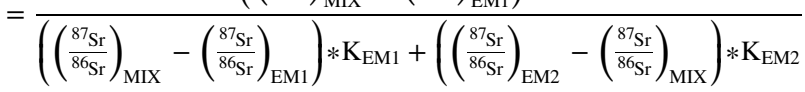

$$
\begin{aligned}
& \% \mathrm{Sr}_{\mathrm{EM} 2}=\frac{\left(\left(\frac{{ }^{87} \mathrm{Sr}}{86 \mathrm{Sr}}\right)_{\mathrm{MIX}}-\left(\frac{{ }^{87} \mathrm{Sr}}{{ }^{86} \mathrm{Sr}}\right)_{\mathrm{EM} 1}\right)}{\left(\left(\frac{87 \mathrm{Sr}}{{ }^{86} \mathrm{Sr}}\right)_{\mathrm{MIX}}-\left(\frac{{ }^{87} \mathrm{Sr}}{{ }^{86} \mathrm{Sr}}\right)_{\mathrm{EM} 1}\right)+\left(\left(\frac{87 \mathrm{Sr}}{{ }^{86} \mathrm{Sr}}\right)_{\mathrm{EM} 2}-\left(\frac{{ }^{87} \mathrm{Sr}}{{ }^{86} \mathrm{Sr}}\right)_{\mathrm{MIX}}\right)}
\end{aligned}
$$

where $\mathrm{K}_{\mathrm{EM} 1}$ and $\mathrm{K}_{\mathrm{EM} 2}$ are $\mathrm{Sr} / \mathrm{Ca}$ molar ratios of end-members 1 and 2, respectively. The carbonate phase from the soil is not taken into account for two main reasons: first, this phase represents only a small amount compared to nodules (the soil carbonate content is $<10 \%$ ); second, this phase is characterized by different features in thin section, compared to nodules (Fig. 3), which can be the result of various late secondary processes, i.e. related to different edaphic conditions. As the ${ }^{87} \mathrm{Sr} /{ }^{86} \mathrm{Sr}$ ratio of carbonate is distinct for each site (Fig. 7), endmember contributions are calculated for each of them (Table 4).

Considering plagioclase and biotite as the two first end-members, calculations result in a Sr contribution of $11 \% \pm 3,9 \% \pm 0.1$, and $10 \% \pm 0.1$ from biotite at sites 1,2 , and 3 , respectively. The calculation error is higher at site 1 , as two profiles have been averaged. Considering the second mixture of plagioclase and Saharan dust, Ca contributions of the atmospheric end-member account for $56 \% \pm 34$, $56 \% \pm 30$, and $24 \% \pm 13$ at sites 1,2 , and 3 , respectively. Amplitudes of errors are essentially due to the estimation of the Sr/ Ca ratio of the Saharan dust. Results of the input calculations are discussed in the next section.

\subsubsection{The Sr/Ca ratio and the potential prominence of in situ weathering}

Chosen end-members and their mixtures do not plot on a mixing line, whether $\mathrm{Sr} / \mathrm{Ca}$ or $1 / \mathrm{Sr}$ is considered (Fig. 4). Sr/Ca and 1/Sr ratios are shifted in carbonate nodules, likely indicating a fractionation during their precipitation. The carbonate nodules have a $\mathrm{Ca} / \mathrm{Sr}$ molar ratio of $1468 \pm 461(\mathrm{n}=16)$ and $6482 \pm 1550(\mathrm{n}=4)$, for site 1 and 2 , and for site 3, respectively (Fig. 4). There are the highest $\mathrm{Ca} / \mathrm{Sr}$ molar ratios of all the samples. Such high $\mathrm{Ca} / \mathrm{Sr}$ ratios are not exceptional, as they have already been observed in carbonate nodules (Violette et al., 2010) and calcretes (Chiquet et al., 1999). Nevertheless, this raises the question about the legitimacy of the input calculations proposed above. Although calculations of the respective contributions take into account the $\mathrm{Sr} / \mathrm{Ca}$ ratio of end-members, it assumes that the dissolution of the two sources is complete and congruent. This could probably be the case for plagioclase, but not for the Saharan dust. Indeed, $\mathrm{Ca}$ is certainly present in various mineral phases of the Saharan dust, such as clays for instance. When $\mathrm{Ca}$ is sorbed on clays, mechanisms leading to its release

Table 4

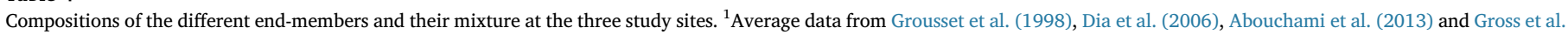

\begin{tabular}{|c|c|c|c|c|c|c|c|}
\hline & $\mathrm{n}$ & $\mathrm{Sr} / \mathrm{Ca}$ molar & & & ${ }^{87} \mathrm{Sr} /{ }^{86} \mathrm{Sr}$ & & \\
\hline Plagioclase on site 1 & 2 & 0.01239 & \pm & 0.00159 & 0.70773 & \pm & 0.00121 \\
\hline Plagioclase on site 2 & 1 & 0.01164 & & & 0.70982 & & \\
\hline Plagioclase on site 3 & 1 & 0.00483 & & & 0.70589 & & \\
\hline Biotite from granite & 1 & n.a. & & & 0.73912 & & \\
\hline Saharan dust average ${ }^{1}$ & 4 , and 8 & 0.00702 & \pm & 0.00374 & 0.71607 & \pm & 0.00136 \\
\hline Carbonate nodules on site 1 & 11 & 0.00077 & \pm & 0.00035 & 0.71121 & \pm & 0.00046 \\
\hline Carbonate nodules on site 2 & 3 & 0.00090 & \pm & 0.00024 & 0.71252 & \pm & 0.00033 \\
\hline Carbonate nodules on site 3 & 4 & 0.00016 & \pm & 0.00005 & 0.70908 & \pm & 0.00006 \\
\hline
\end{tabular}
(2016). 
in the soil solution are different from those acting in the case of plagioclases. Therefore, the calculations of contributions proposed in Section 5.3.1 must be considered as a theoretical approach as they assume that both sources are dissolved in the same way: results must therefore be considered with caution.

However, regarding results of the contribution calculations, it is interesting to note that the input of plagioclase is dominant at site 3 , where the bedrock is rich in Ca. Moreover, the Ca contribution of the Saharan dust seems to be related to its abundance in the CRPM. Indeed, the Ca contribution of Saharan dust and plagioclases are equivalent in the granitic area, where the CRPM is constituted by about $50 \%$ of Saharan dust and granite weathered products (Fig. 7). In contrast, in the greenstone area, the fraction of Saharan dust in the CRPM is low, and thus their contribution to the Ca supply is also low. Regarding biotite, only a small amount is needed to shift the ${ }^{87} \mathrm{Sr} /{ }^{86} \mathrm{Sr}$ ratio of the carbonate nodule values. The ${ }^{87} \mathrm{Sr} /{ }^{86} \mathrm{Sr}$ of carbonate nodules likely results from a mixture of the three end-members (i.e. plagioclases, biotite, and Saharan dust). If only a small amount of $\mathrm{Sr}$ is provided by biotite (less than a few percent, which seems reasonable), therefore the Ca contribution from the Saharan dust will decrease significantly, making plagioclase the main Ca source. Consequently, it is possible to conclude that the Ca contribution of in situ weathering to carbonate nodules is significant in the granitic and greenstone areas of Far North Region of Cameroon.

5.4. What mechanisms influence the respective contributions of the in situ weathering and the atmospheric inputs to the Ca supply?

The present study demonstrated that the Ca contribution from in situ weathering is significant, which is different from many other studies emphasizing a dominance of atmospheric inputs (i.e. Quade et al., 1995; Capo and Chadwick, 1999; Chiquet et al., 1999; Hamidi et al., 2001; Van der Hoven and Quade, 2002; Rech et al., 2003; Lintern et al., 2006; Dart et al., 2007; Huerta et al., 2015). Only three studies reported a dominance of in situ weathering i.e. Rech et al. (2003) in the Atacama Desert, and Durand et al. (2006) and Violette et al. (2010) in India. At this stage, a question arises: what influences the respective contributions of the in situ weathering and the atmospheric inputs to the Ca supply? Violette et al. (2010) proposed that the higher the mean annual rainfall, the more significant the contribution of in situ weathering. However, it seems dubious to establish a direct relationship between the mean annual rainfall and the contribution of the in situ weathering. Indeed, ages of the secondary carbonates reported in the different studies cited above are unknown, meaning they can be inherited features. Thus, it does not seem reasonable to compare potentially inherited features with present-day mean annual rainfalls. Nevertheless, high mean annual rainfalls impact in situ weathering, and consequently, the release of $\mathrm{Ca}$ in the soil solution. The important contribution of in situ weathering at the Far North Region of Cameroon is consistent with the fact that the carbonate nodules are inherited from the African Humid Period (Diaz et al., 2016b) during which, rainfall was much higher than present-day.

A second parameter, which can potentially have an influence on the in situ weathering contribution, is the distance from the coastline. Indeed, close to the coastline, the sea spray can also contribute to Ca supply and its contribution seems to decrease in proportion to the distance from the coastline (Dart et al., 2007). However, in remote hinterlands, dust has a much more important impact than sea spray. For instance, in Morocco, Hamidi et al. (2001) stated that dust is the main Ca source of secondary carbonates observed on a Ca-rich bedrock, $200 \mathrm{~km}$ inland. In the Canaries Island, Huerta et al. (2015) studied secondary carbonates on Ca-rich bedrocks close to the coastline and they considered dust as the main Ca source instead of sea spray. In the Far North Region of Cameroon, $1000 \mathrm{~km}$ inland, the present study emphasized the importance of the in situ weathering, especially on Carich bedrock. The prominence of in situ weathering in the Ca-poor bedrock settings of Far North Region of Cameroon can be explained by the relative preservation of plagioclases in soils. Indeed, Vertisols associated with carbonate nodules are known to be alkaline and enriched in 2:1 clays (Coulombe et al., 1996), i.e. favourable to primary mineral preservation. It is remarkable to note that in two other studies, which concluded a dominant contribution from in situ weathering (Durand et al., 2006; Violette et al., 2010), soils are similar to those in the Far North Region of Cameroon (presence of some alkalinity and 2:1 clays), and thus supporting the preservation of primary Ca-phases. This conclusion highlights the role of edaphic conditions as significant factors in regulating the contribution of in situ weathering in calcium dynamics of landscapes. Hence, in these peculiar settings, an important part of the Ca seems to be simply transferred from bedrock to carbonate nodules.

\section{Conclusions}

In the Far North Region of Cameroon, soil Ca-carbonate and Cacarbonate nodules originate from the same Ca sources. The carbonate phases of three different study sites display a different $\mathrm{Sr}$ isotopic composition, indicating that a local source participated to the $\mathrm{Ca}$ supply. This local source is the plagioclases, as they show co-variations in their Sr isotopic composition with carbonate phases and as demonstrated by calculations of the respective contributions. A second source, more radiogenic, is needed to explain the $\mathrm{Sr}$ signature of these different carbonate phases. The Saharan dust and biotite seems to be the best candidates, as they partly contributed to the CRPM composition, making the latter a mixture of aeolian particles and weathered products from bedrocks. The $\mathrm{Ca} / \mathrm{Sr}$ ratio is not conservative during carbonate nodule precipitation and the reasons for that are still unclear. Thus, calculations of contributions from each source are theoretical with some limits. Nevertheless, the weathered local bedrock (granite and greenstone) remains a significant Ca source. This prominence of a local contribution is quite surprising, as many authors have reported the dominance of allochthonous inputs, such as dust. When compared with other studies, the preservation of primary Ca-phases in the soils of the Far North Region of Cameroon remains a key factor in promoting a significant contribution of in situ weathering to the Ca supply for carbonate nodules.

\section{Acknowledgements}

The authors want to thank Dr. Jean-Louis Rajot and the other members of the Hydraride program (2011), who drew our attention to the mounds of the Sudano-Sahelian belt and collected the first samples from Niger and Burkina Faso. They also made the first observations in Northern Cameroon. The PEERS MORDRED insured the logistical aspects during the initial fieldwork in 2012. The LMI PICASS'EAU from the IRD and the members of Water and Environmental Sciences Laboratory at the University of Ngaoundéré provided their help for logistical and technical support for fieldwork in 2013. Dr. Thierry Adatte (ISTE, University of Lausanne) performed XRD analyses, Dr. Alexey Ulianov (ISTE, University of Lausanne) performed ICP-MS analysis, Dr. Massimo Chiaradia (Section of Earth Environmental Science, University of Geneva) and Dr. Abel Guihou (CEREGE, University Aix-Marseille) performed $\mathrm{Sr}$ and $\mathrm{Nd}$ measurements. The authors would like to thank the two anonymous reviewers who helped them to improve the paper content. Karin Verrecchia edited the English version of the manuscript. PD is supported by the Agence Nationale de la Recherche through the EQUIPEX ASTER-CEREGE Project and the LABEX OT-MED.This study has been supported by the Swiss National Science Foundation (grant no. 200021-147038 and 159901).

\section{Appendix A. Supplementary data}

Supplementary data to this article can be found online at http://dx. 
doi.org/10.1016/j.chemgeo.2017.04.015.

\section{References}

Abouchami, W., et al., 2013. Geochemical and isotopic characterization of the Bodélé depression dust source and implications for transatlantic dust transport to the Amazon Basin. Earth Planet. Sci. Lett. 380, 112-123.

Arunchalam, J., Emons, H., Krasnodebska, B., Mohl, C., 1996. Sequential extraction studies on homogenized forest soil samples. Sci. Total Environ. 181, 147-159.

Blum, J.D., Erel, Y., 1997. Rb-Sr isotope systematics of a granitic soil chronosequence: the importance of biotite weathering. Geochim. Cosmochim. Acta 61 (15), 3193-3204.

Bocquier, G., 1973. Genèse et évolution de deux toposéquences de sols tropicaux du Tchad: interprétation biogéodynamique. In: Mémoires de l'ORSTOM, Paris, France, pp. 327.

Borg, L.E., Banner, J.L., 1996. Neodymium and strontium isotopic constraints on soil sources in Barbados, West Indies. Geochim. Cosmochim. Acta 60 (21), 4193-4206.

Bory, A., 1997. Etude des flux de matériel terrigène dans la colonne d'eau de l'Atlantique subtropical nord-est. In: Relations avec les apports atmosphériques, .

Bowen, H.J.M., 1979. Environmental Chemistry of the Elements. Academic Press.

Brabant, P., Gavaud, M., 1985. Les sols et les ressources en terres du Nord-Cameroun. In: ORTSTOM, notice explicative, 103, pp. 369.

Capo, R.C., Chadwick, O.A., 1999. Sources of strontium and calcium in desert soil and calcrete. Earth Planet. Sci. Lett. 170 (1), 61-72.

Capo, R.C., Stewart, B.W., Chadwick, O.A., 1998. Strontium isotopes as tracers of ecosystem processes: theory and methods. Geoderma 82 (1-3), 197-225.

Cerling, 1984. The stable isotopic composition of modern soil carbonate and its relationship to climate. Earth Planet. Sci. Lett. 71, 229-240.

Chiquet, A., Michard, A., Nahon, D., Hamelin, B., 1999. Atmospheric input vs in situ weathering in the genesis of calcretes: an $\mathrm{Sr}$ isotope study at Galvez (Central Spain). Geochim. Cosmochim. Acta 63 (3-4), 311-323.

Clauer, N., 1981. Strontium and argon isotopes in naturally weathered biotites, muscovites and feldspars. Chem. Geol. 31, 325-334.

Clauer, N., Chaudhuri, S., 1995. Clays in crustal environments. In: Isotope Tracing and Dating. Springer-Verlag, Berlin.

Cole, J.M., Goldstein, S.L., deMenocal, P.B., Hemming, S.R., Grousset, F.E., 2009. Contrasting compositions of Saharan dust in the eastern Atlantic Ocean during the last deglaciation and African Humid Period. Earth Planet. Sci. Lett. 278 (3-4), 257-266.

Coulombe, C., Dixon, J., Wilding, L., Ahmad, N., Mermut, A., 1996. Mineralogy and chemistry of vertisols. In: Vertisols and Technologies for Their Management, pp. 115-200.

Cramer, M.D., Barger, N.N., 2014. Are mima-like mounds the consequence of long-term stability of vegetation spatial patterning? Palaeogeogr. Palaeoclimatol. Palaeoecol. 409, 72-83.

Crouvi, O., Amit, R., Enzel, Y., Gillespie, A.R., 2010. Active sand seas and the formation of desert loess. Quat. Sci. Rev. 29 (17-18), 2087-2098.

Dart, R.C., Barovich, K.M., Chittleborough, D.J., Hill, S.M., 2007. Calcium in regolith carbonates of central and southern Australia: its source and implications for the global carbon cycle. Palaeogeogr. Palaeoclimatol. Palaeoecol. 249 (3-4), 322-334.

Derry, L.A., Chadwick, O.A., 2007. Contributions from Earth's atmosphere to soil. Elements 3 (5), 333-338.

Dia, A., Chauvel, C., Bulourde, M., Gérard, M., 2006. Eolian contribution to soils on Mount Cameroon: isotopic and trace element records. Chem. Geol. 226 (3), 232-252.

Diaz, N., et al., 2016a. Can mima-like mounds be vertisol relics (Far North Region of Cameroon, Chad Basin)? Geomorphology 261, 41-56.

Diaz, N., et al., 2016b. A 20-ka reconstruction of a Sahelo-Sudanian paleoenvironment using multi-method dating on pedogenic carbonate. In: EGU General Assembly Conference Abstracts, pp. 4243.

Dupré, B., Négrel, P., Seimbille, F., Allègre, J., 1994. 87Sr/S6Sr ratio variation during a rain event. Atmos. Environ. 28 (4), 617-620.

Durand, N., Ahmad, S., Hamelin, B., Gunnell, Y., Curmi, P., 2006. Origin of Ca in South Indian calcretes developed on metamorphic rocks. J. Geochem. Explor. 88 (1), 275-278.

Elbersen, G., Davies, G., Van Reeuwijk, L., Janssen, P., 2000. Quantification of the incorporation of atmospheric $\mathrm{CO}_{2}$ in calcific horizons. In: Global Climate Change and Pedogenic Carbonates. Lewis Publishers, Boca Raton, Florida, pp. 87-95.

Engelstaedter, S., Tegen, I., Washington, R., 2006. North African dust emissions and transport. Earth Sci. Rev. 79 (1), 73-100.

Erhart, M.H., 1954. Bassin du Moyen Logone. In: Commission Scientifique du Logone et du Tchad, Etude Pédologique du Bassin Alluvionnaire du Logone-Chari. ORSTOM, Paris, France, pp. 17-107.

Faure, G., Powell, J.L., 2012. Strontium Isotope Geology, 5. Springer Science \& Business Media.

Gazel, J., Guiraudie, C., de Ribes, G.C., Cirotteau, A., Leroy, B., 1956. Carte géologique du Cameroun. Direction des mines et de la geologie du Cameroun.

Gross, A., Palchan, D., Krom, M., Angert, A., 2016. Elemental and isotopic composition of surface soils from key Saharan dust sources. Chem. Geol. 442, 54-61.

Grousset, F.E., Biscaye, P.E., 2005. Tracing dust sources and transport patterns using Sr, $\mathrm{Nd}$ and $\mathrm{Pb}$ isotopes. Chem. Geol. 222 (3), 149-167.

Grousset, F., Rognon, P., Coudé-Gaussen, G., Pédemay, P., 1992. Origins of peri-Saharan dust deposits traced by their $\mathrm{Nd}$ and $\mathrm{Sr}$ isotopic composition. Palaeogeogr. Palaeoclimatol. Palaeoecol. 93 (3), 203-212.

Grousset, F., et al., 1998. Saharan wind regimes traced by Sr-Nd isotopic composition of subtropical atlantic sediments: last glacial maximum vs today. Quat. Sci. Rev. 17, 395-409.
Hamidi, E.M., Colin, F., Michard, A., Boulange, B., Nahon, D., 2001. Isotopic tracers of the origin of $\mathrm{Ca}$ in a carbonate crust from the Middle Atlas, Morocco. Chem. Geol. 176 (1-4), 93-104.

Hasinger, O., et al., 2015. Carbon dioxide in scree slope deposits: a pathway from atmosphere to pedogenic carbonate. Geoderma 247, 129-139.

Hervieu, J., 1967. Sur l'existence de deux cycles climato-sédimentaires quaternaires dans les monts Mandara et leurs abords (Nord-Cameroun). Conséquences morphologiques et pédogénétiques. In: Comptes Rendus de l'Académie des Sciences, Paris, 264, pp. 2624-2627.

Huerta, P., et al., 2015. The role of climate and aeolian dust input in calcrete formation in volcanic islands (Lanzarote and Fuerteventura, Spain). Palaeogeogr. Palaeoclimatol. Palaeoecol. 417, 66-79.

Jacobsen, S.B., Wasserburg, G., 1980. Sm-Nd isotopic evolution of chondrites. Earth Planet. Sci. Lett. 50 (1), 139-155.

Kalu, A., 1979. The African dust plume: its characteristics and propagation across West Africa in winter. Scope 14, 95-118.

Khadkikar, A., Merh, S., Malik, J., Chamyal, L., 1998. Calcretes in semi-arid alluvial systems: formative pathways and sinks. Sediment. Geol. 116 (3), 251-260.

Kovda, I., Mora, C.I., Wilding, L.P., 2006. Stable isotope compositions of pedogenic carbonates and soil organic matter in a temperate climate vertisol with gilgai, southern Russia. Geoderma 136 (1), 423-435.

Lal, R., Kimble, J.M., Stewart, B., Eswaran, H., 1999. Global Climate Change and Pedogenic Carbonates. Lewis Publishers, United States, pp. 305.

Lamotte, M., Bruand, A., Humbel, F.X., Herbillon, A.J., Rieu, M., 1997. A hard sandyloam soil from semi-arid northern Cameroon: 1. Fabric of the groundmass. Eur. J. Soil Sci. 48 (2), 213-225.

Likens, G., et al., 1998. The biogeochemistry of calcium at Hubbard Brook. Biogeochemistry 41 (2), 89-173.

Lintern, M., Sheard, M., Chivas, A., 2006. The source of pedogenic carbonate associated with gold-calcrete anomalies in the western Gawler Craton, South Australia. Chem. Geol. 235 (3), 299-324.

Maley, J., 1981. Etudes Paylnologiques dans le Bassin du Tchad et Paléoclimatologie de l'Afrique Nord-Tropicale de 30000 ans à l'Epoque Actuelle. In: Paleoecology of Africa, 13, pp. 45-52.

Martin, D., 1961. Carte pédologique du Nord-Cameroun au 1/100000e feuille Mora. ORSTOM-IRCAM, Yaoundé, Cameroun.

McLennan, S., Hemming, S., Mc Daniel, D., Hanson, G., 1993. Geochemical approaches to sedimentation, provenance, and tectonics. In: Geological Society of America, Special Paper 284, .

de Menocal, P., et al., 2000. Abrupt onset and termination of the African Humid Period: rapid climate responses to gradual insolation forcing. Quat. Sci. Rev. 19 (1-5), 347-361.

Morin, S., 2000. Géomorphologie. In: Seignobos, C., Iyébi-Mandjek, O. (Eds.), Atlas de la Province Extrême-Nord Cameroun. Ministère de la Recherche Scientifique et Technique, Institut National de Cartographie, Institut de Recherche pour le Développement, Cameroun, Paris, France, pp. 49.

Mpeck, E., 1994. Contribution des poussières sahariennes à la sédimentation du pleistocène supérieur au nord Cameroun: la formation de Doyang. pp. 303.

Negrel, P., Allègre, C.J., Dupré, B., Lewin, E., 1993. Erosion sources determined by inversion of major and trace element ratios and strontium isotopic ratios in river water: the Congo Basin case. Earth Planet. Sci. Lett. 120 (1-2), 59-76.

Négrel, P., Guerrot, C., Millot, R., 2007. Chemical and strontium isotope characterization of rainwater in France: influence of sources and hydrogeochemical implications. Isot. Environ. Health Stud. 43 (3), 179-196.

Olivry, J., Naah, E., 1999. Hydrologie. In: Atlas de la Province Extrême-Nord Cameroun, Paris, Planche 3, pp. 32.

Pearce, C.R., Parkinson, I.J., Gaillardet, J., Chetelat, B., Burton, K.W., 2015 Characterising the stable ( $888 / 86 \mathrm{Sr}$ ) and radiogenic $(87 \mathrm{Sr} / 86 \mathrm{Sr})$ isotopic composition of strontium in rainwater. Chem. Geol. 409, 54-60.

Pelt, E., et al., 2013. Atmospheric dust contribution to the budget of U-series nuclides in soils from the Mount Cameroon volcano. Chem. Geol. 341, 147-157.

Pett-Ridge, J.C., Derry, L.A., Barrows, J.K., 2009. Ca/Sr and 87Sr/86Sr ratios as tracers of $\mathrm{Ca}$ and Sr cycling in the Rio Icacos watershed, Luquillo Mountains, Puerto Rico. Chem. Geol. 267 (1-2), 32-45.

Pias, J., 1962. Les Sols du Moyen et Bas Logone, du Bas Chari, des Régions Riveraines du Lac Tchad et du Bahr El Ghazal. In: Mémoires ORSTOM, Paris, France, pp. 434.

Pin, C., Briot, D., Bassin, C., Poitrasson, F., 1994. Concomitant separation of strontium and samarium-neodymium for isotopic analysis in silicate samples, based on specific extraction chromatography. Anal. Chim. Acta 298 (2), 209-217.

Quade, J., Chivas, A.R., Mcculloch, M.T., 1995. Strontium and carbon-isotope tracers and the origins of soil carbonate in South-Australia and Victoria. Palaeogeogr. Palaeoclimatol. Palaeoecol. 113 (1), 103-117.

Rech, J.A., Quade, J., Hart, W.S., 2003. Isotopic evidence for the source of Ca and S in soil gypsum, anhydrite and calcite in the Atacama Desert, Chile. Geochim. Cosmochim. Acta 67 (4), 575-586.

Schlesinger, W.H., 1982. Carbon storage in the caliche of arid soils: a case study from Arizona. Soil Sci. 133 (4), 247-255.

Schwanghart, W., Schütt, B., 2008. Meteorological causes of Harmattan dust in West Africa. Geomorphology 95 (3-4), 412-428.

Sieffermann, G., 1967. Variations Climatiques au Quaternaire dans le Sud-Ouest de la Cuvette Tchadienne. In: Fonds Documentaires ORSTOM, 141(6), pp. 12.

Skonieczny, C., et al., 2011. The 7-13 March 2006 major Saharan outbreak: Multiproxy characterization of mineral dust deposited on the West African margin. J. Geophys. Res. Atmos. 116 (1984-2012). (D18).

Suchel, J.-B., 1988. Les climats du Cameroon. Université de St-Etienne, France, pp. 188.

Van der Hoven, S.J., Quade, J., 2002. Tracing spatial and temporal variations in the 
sources of calcium in pedogenic carbonates in a semiarid environment. Geoderma 108 (3), 259-276.

Verrecchia, E.P., 2011. Pedogenic carbonates. In: Encyclopedia of Geobiology, pp. 721-725.

Violette, A., et al., 2010. Formation and preservation of pedogenic carbonates in South India, links with paleo-monsoon and pedological conditions: clues from $\mathrm{Sr}$ isotopes, U-Th series and REEs. Geochim. Cosmochim. Acta 74 (24), 7059-7085.
White, A.F., Blum, A.E., 1995. Effects of climate on chemical weathering in watersheds. Geochim. Cosmochim. Acta 59 (9), 1729-1747.

Wieder, M., Yaalon, D., 1982. Micromorphological fabrics and developmental stages of carbonate nobular forms related to soil characteristics. Geoderma 28 (3), 203-220. Zamanian, K., Pustovoytov, K., Kuzyakov, Y., 2016. Pedogenic carbonates: forms and formation processes. Earth Sci. Rev. 157, 1-17. 